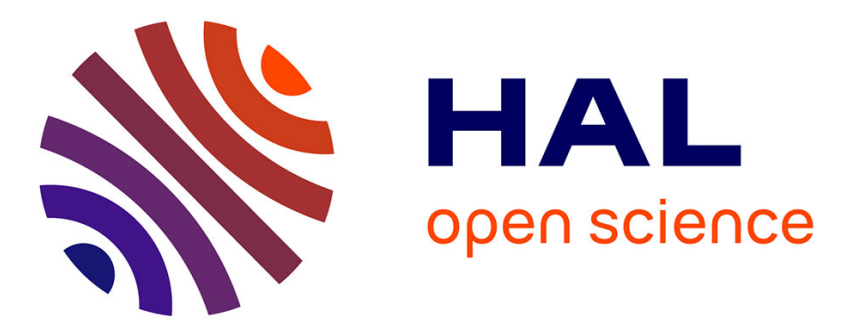

\title{
Equatorially asymmetric convection inducing a hemispherical magnetic _eld in rotating spheres and implications for the past martian dynamo
}

Maylis Landeau, Julien Aubert

\section{- To cite this version:}

Maylis Landeau, Julien Aubert. Equatorially asymmetric convection inducing a hemispherical magnetic _eld in rotating spheres and implications for the past martian dynamo. Physics of the Earth and Planetary Interiors, 2011, 185 (3-4), pp.61. 10.1016/j.pepi.2011.01.004 . hal-00735849

\author{
HAL Id: hal-00735849 \\ https://hal.science/hal-00735849
}

Submitted on 27 Sep 2012

HAL is a multi-disciplinary open access archive for the deposit and dissemination of scientific research documents, whether they are published or not. The documents may come from teaching and research institutions in France or abroad, or from public or private research centers.
L'archive ouverte pluridisciplinaire HAL, est destinée au dépôt et à la diffusion de documents scientifiques de niveau recherche, publiés ou non, émanant des établissements d'enseignement et de recherche français ou étrangers, des laboratoires publics ou privés. 


\section{Accepted Manuscript}

Title: Equatorially asymmetric convection inducing a

hemispherical magnetic _eld in rotating spheres and implications for the past martian dynamo

Authors: Maylis Landeau, Julien Aubert

PII:

S0031-9201(11)00014-8

DOI: doi:10.1016/j.pepi.2011.01.004

Reference: PEPI 5372

To appear in: $\quad$ Physics of the Earth and Planetary Interiors

Received date: $\quad$ 23-6-2010

Revised date: $\quad 23-11-2010$

Accepted date: $\quad$ 16-1-2011

Please cite this article as: Landeau, M., Aubert, J., Equatorially asymmetric convection inducing a hemispherical magnetic eld in rotating spheres and implications for the past martian dynamo, Physics of the Earth and Planetary Interiors (2008), doi:10.1016/j.pepi.2011.01.004

This is a PDF file of an unedited manuscript that has been accepted for publication. As a service to our customers we are providing this early version of the manuscript. The manuscript will undergo copyediting, typesetting, and review of the resulting proof before it is published in its final form. Please note that during the production process errors may be discovered which could affect the content, and all legal disclaimers that apply to the journal pertain. 


\section{Abstract}

The convective instability in a rapidly rotating, self-graviting sphere sets up in the form of equatorially symmetric, non-axisymmetric columnar vortices aligned with the rotation axis, carrying heat away in the cylindrical radial direction. In this study, we present numerical simulations of thermal convection and dynamo action driven by internal heating (intended to model a planetary core subject to uniform secular cooling) in a rotating sphere where, from the classical columnar convection regime, we find a spontaneous transition towards an unexpected and previously unobserved flow regime in which an equatorially antisymmetric, axisymmetric (EAA) mode strongly influences the flow. This EAA mode carries heat away along the rotation axis and is the nonlinear manifestation of the first linearly unstable axisymmetric mode. When the amplitude of the EAA mode reaches high enough values, we obtain hemispherical dynamos with one single hemisphere bearing more than 75 percent of the total magnetic energy at the surface of the rotating sphere. We perform the linear analysis of the involved convective modes and the nonlinear study of this hydrodynamic transition, with and without dynamo action, to obtain scaling laws for the regime boundaries. As secular cooling in a full sphere (i.e. without inner core) is a configuration which has probably been widespread in the early solar system in planetary cores, including the core of Mars, we discuss the possible implications of our results for the past martian dynamo.

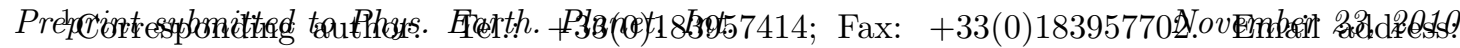
landeau@ipgp.fr

\section{Introduction}

Convection in rotating systems has been widely studied because of its numerous geophysical and astrophysical applications. For instance, dynamo processes sus- 
tained by convection are an attractive explanation not only for the Sun's magnetic field but also for the magnetic field of the Earth and other planets. Rotationally dominated convection is typically organized into vortices aligned with the rotation axis. These columnar structures tend not to violate the Taylor-Proudman constraint which requires the velocity field to be invariant along any line parallel to the rotation axis and which is approximately valid when the main balance is between the Coriolis force and the pressure gradient force. In the particular case of rotating spheres, the idea of a columnar convection appeared gradually. The first attempts to solve the onset of thermal convection focused on axisymmetric modes. Scaling laws for the threshold of instability of these modes could be extracted from Chandrasekhar (1961), but the asymptotic behavior in the limit of small Ekman numbers was obtained by Roberts (1965) and Bisshopp and Niiler (1965) with two different analytical approaches. Roberts (1968) was the first to recognize that the important modes at the onset of thermal convection in rapidly rotating spheres are non-axisymmetric. However, Roberts concentrated his efforts on equatorially antisymmetric modes, in the wake of his 1965 study (Roberts, 1965) where he found that the linearly most unstable axisymmetric mode of convection has this parity. Busse (1970) subsequently showed that the dominant structures at onset are not only non-axisymmetric but also equatorially symmetric, corresponding to the famous illustration of vortices parallel to the axis of rotation and localized in the vicinity of a fixed radius in cylindrical coordinates. The first correct linear asymptotic solution for rapidly rotating full spheres was given by Jones et al. (2000). Nonlinear numerical simulations of convection and dynamo action in spherical shells have subsequently confirmed this columnar flow structure and the secondary influence of equatorially antisymmetric modes (e.g. Olson et al., 1999).

Among the different driving mechanisms which can be imposed in such numerical simulations, secular cooling in full spheres (i.e. without inner core) has been studied little until now. This configuration is appropriate for modeling convection and dynamo action in the Earth's core prior to inner core nucleation (Gubbins et al., 2003; Aubert et al., 2009). Besides, an early dynamo in a convective core subject to secular cooling is the most plausible hypothesis to explain the strong magnetizations measured on Mars' crust by the Mars Global Surveyor mission. The timing of the martian dynamo is debated but can be estimated using ages of the different crust regions. Indeed, some large impact basins, believed to be $\sim 4$ Gyr old, are not magnetized (Acuna et al., 1999). Thus, the dynamo would have been active in the early history of Mars, between 4.5 Gyr and 4 Gyr. Several 
published studies (Lodders and Fegley, 1997; Sanloup et al., 1999) compared sulphur contents of martian meteorites with those of other primitive meteorites and estimated a high sulphur content in Mars' core: from $10.6 \%$ to $16.2 \%$. Stewart et al. (2007) performed experiments on iron-sulfur and iron-nickel-sulfur systems at high pressure and obtained the corresponding phase diagrams at fixed pressure. They showed that, considering such high sulphur contents, Mars' core is likely to be presently entirely liquid.

The Mars Global Surveyor mission also revealed a very surprising feature for Mars' crust: intense crustal magnetizations were measured in the Southern hemisphere whereas the Northern hemisphere contains only weak fields. Dynamo models do not easily explain this hemispherical crustal magnetic field. Since Mars is a terrestrial planet with a size comparable to that of the Earth, we could have expected a dipole dominated dynamo regime with similar magnetic field strength in both hemispheres. For this reason it has long been thought that post-dynamo events, such as resurfacing processes or giant impacts, were responsible for the magnetic field asymmetry of the martian crust. It is however possible (Stanley et al., 2008) that hemispherical magnetizations of Mars' surface have been caused by a dynamo process, influenced by a hemispherical pattern in the heat flux extracted by the mantle at the core-mantle boundary (CMB).

Here, we use numerical simulations to model thermal convection and dynamo action driven by secular cooling in rotating full spheres. We find that, in this geometry and with this driving mechanism, an unexpected and previously unobserved flow regime spontaneously emerges through a hydrodynamic bifurcation: from the classical columnar flow regime to a flow regime which is strongly influenced by an equatorially antisymmetric, axisymmetric (EAA) mode and which apparently violates the Taylor-Proudman constraint. This unexpected flow regime, which we will refer to as the asymmetric regime, has never been observed before. The aim of the present study is to investigate the following questions: What is the dynamics of this EAA mode and why does it appear in the particular case of convection driven by secular cooling in rotating spheres? What impact does the EAA mode have on the pattern of magnetic field which can be seen on the planetary surface? In section 2 we present the model and the equations solved by the numerical code. In section 3 we introduce the results related to the hydrodynamics of the system. In section 4 we analyze the effect of the emergence of the EAA mode on magnetic field generation and we show that hemispherical dynamos can be spontaneously induced. Finally, in section 5, we discuss our numerical results and the possible implications for the past martian dynamo. 


\section{Model}

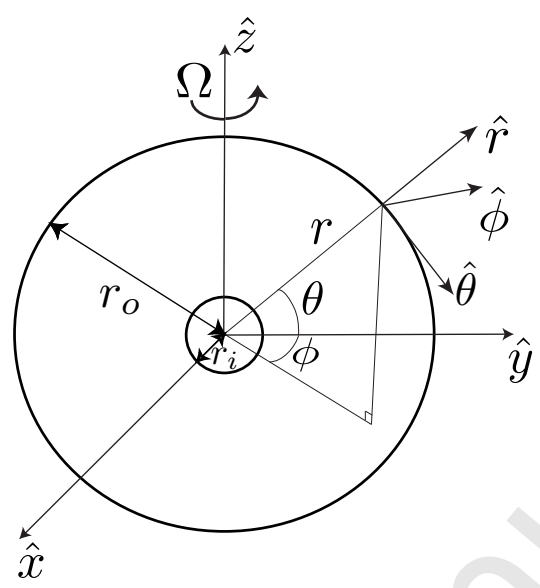

Figure 1: Schematic representation of the system. $r_{i} / r_{o}=0.01$.

Fig.1 illustrates the configuration of the system. We use spherical coordinates $(r, \theta, \phi)$ and cylindrical coordinates $(s, \phi, z)$. A sphere of radius $r_{o}$, which contains a conductive fluid, is rotating at rate $\Omega$ around an axis parallel to $\hat{z}$. Because of numerical considerations, for the calculations performed in this study we retained a very small inner sphere of radius $r_{i}=0.01 r_{o}$ at the center of the system. It has already been argued (Aubert et al., 2009) that the presence of the small inner sphere has a negligible impact on the solution. After implementation of a more recent version of our code where the inner sphere is completely removed $\left(r_{i} / r_{o}=0\right)$, we were able to confirm that this is indeed the case for the results presented here. For this reason, the system will be referred to as a rotating full sphere.

Within the magnetohydrodynamic approximation, the non-dimensionalized governing Boussinesq equations for the velocity field $\mathbf{u}$, the magnetic field $\mathbf{B}$, and the temperature field $T$, are given by:

$$
\begin{gathered}
\frac{\partial \mathbf{u}}{\partial t}+\mathbf{u} . \nabla \mathbf{u}+2 \hat{z} \times \mathbf{u}=-\nabla P+R a_{Q} \frac{\mathbf{r}}{r_{0}} T+(\nabla \times \mathbf{B}) \times \mathbf{B}+E \Delta \mathbf{u} \\
\frac{\partial T}{\partial t}+(\mathbf{u} . \nabla) T=\frac{E}{P r} \Delta T+S_{T}
\end{gathered}
$$




$$
\frac{\partial \mathbf{B}}{\partial t}=\nabla \times(\mathbf{u} \times \mathbf{B})+\frac{E}{P m} \Delta \mathbf{B}
$$

$$
\begin{aligned}
\nabla \cdot \mathbf{B} & =0 \\
\nabla \cdot \mathbf{u} & =0
\end{aligned}
$$

where $S_{T}$ is a positive source term. The equations have been non-dimensionalized using the following scales: $D=r_{o}-r_{i}$ for length scale $\left(D \approx r_{o}\right), \Omega^{-1}$ for time, $\Omega D$ for velocity, $\rho D^{2} \Omega^{2}$ for pressure where $\rho$ is the fluid density, $\sqrt{\rho \mu} \Omega D$ for magnetic field where $\mu$ is the magnetic permeability of the fluid and $Q / 4 \pi \rho C_{p} \Omega D^{3}$ for temperature where $Q$ is the total heat flux at the external boundary, or CMB and $C_{p}$ the specific heat capacity.

Our numerical code solves the Boussinesq equations (1)-(5) for a system which corresponds to fluctuations with respect to an adiabatic reference state. In this framework, we model secular cooling in planetary systems using internal heating in the Boussinesq system. The decrease in the adiabatic (reference) temperature on geological time scales is modeled by a uniform distribution of internal heat sources $\left(S_{T}\right)$ in equation (2). As $T$ has to be statistically stationary, $S_{T}$ is determined such that the heat budget of the sphere vanishes (Aubert et al., 2009).

The mantle dynamics evolves on much longer time scales than the core dynamics and thus, the core provides an isothermal boundary condition for the mantle. The resulting heat flux at the CMB, either related to thermal boundary layers in a convective mantle or to a conductive heat flux in a stagnant mantle, provides the thermal boundary condition for core convection. Thus, we impose a uniform heat flux $Q$ at the surface of the sphere which represents the CMB. The heat flux is equal to zero at $r_{i}$. The velocity vanishes on the rigid boundaries. We study hydrodynamic simulations (in which the initial magnetic field is set to zero) and dynamo simulations (in which the initial magnetic field corresponds to a dipole of infinitesimal amplitude).

Non-dimensional control parameters are:

- the modified Rayleigh number

$$
R a_{Q}=\frac{\alpha g_{0} Q}{4 \pi \rho C_{p} \Omega^{3} D^{4}},
$$

which has the advantage of being independent of the thermal and viscous diffusivities (Christensen and Aubert, 2006; Aubert et al., 2009), 
- the Ekman number

$$
E=\frac{\nu}{\Omega D^{2}}
$$

- the Prandtl number

$$
\operatorname{Pr}=\frac{\nu}{\kappa}
$$

- the magnetic Prandtl number

$$
P m=\frac{\nu}{\eta}
$$

where $\alpha$ is the thermal expansion coefficient, $g_{o}$ is the acceleration due to gravity at the outer radius, $\nu$ the kinematic viscosity, $\kappa$ the thermal diffusivity and $\eta$ the magnetic diffusivity. Using this choice of non-dimensional numbers, the canonical Rayleigh number $R a$ is given by $R a=R a_{Q} E^{-3} \operatorname{Pr}^{2}$.

The numerical code PARODY is used to solve the entire set of nonlinear equations (1-5). More details about this code can be found in Aubert et al. (2008). The parameters of all the nonlinear simulations used in this study are contained in Table 1 (hydrodynamic simulations) and Table 2 (dynamo simulations): we vary the values of $E$ and $R a_{Q}$ and set $\operatorname{Pr}$ to 1 and $P m$ to 5 in most simulations. Linear stability results are obtained using a linear version of PARODY. The equations (1-5) are linearized in order to get the corresponding perturbation equations. The basic state corresponds to a stagnant fluid in which heat is transferred by diffusive processes. The algorithm used here is the same as in Dormy et al. (2004): it does not solve an eigenvalue problem but, for each value of the modified Rayleigh number, it integrates the equations in time until the system converges towards a given eigenfunction of the form $F(r) \exp (\sigma t) \exp i(m \phi-\omega t)$ for each azimuthal wavenumber $m$. Then, we increase the Rayleigh number until the growth rate of a particular mode with azimuthal wavenumber $m_{c}$ becomes positive. As for the nonlinear analysis, we set $\operatorname{Pr}=1$ and we vary the Ekman and modified Rayleigh numbers.

As the results presented in this study are rather unexpected, special care has been devoted to testing our numerical implementation PARODY against at least another implementation (the Christensen, Wicht, Glatzmaier MAG/MAGIC code, Christensen et al., 2001) in a case where antisymmetric convection arises in the presence of an inner core, with the following parameters: $E=10^{-4}, R a_{Q}=2 \cdot 10^{-4}$, $\operatorname{Pr}=1, \operatorname{Pm}=7$, and an aspect ratio $r_{i} / r_{o}=0.35$. We have checked that after equilibration, both codes yield the same results, with an equatorially asymmetric 


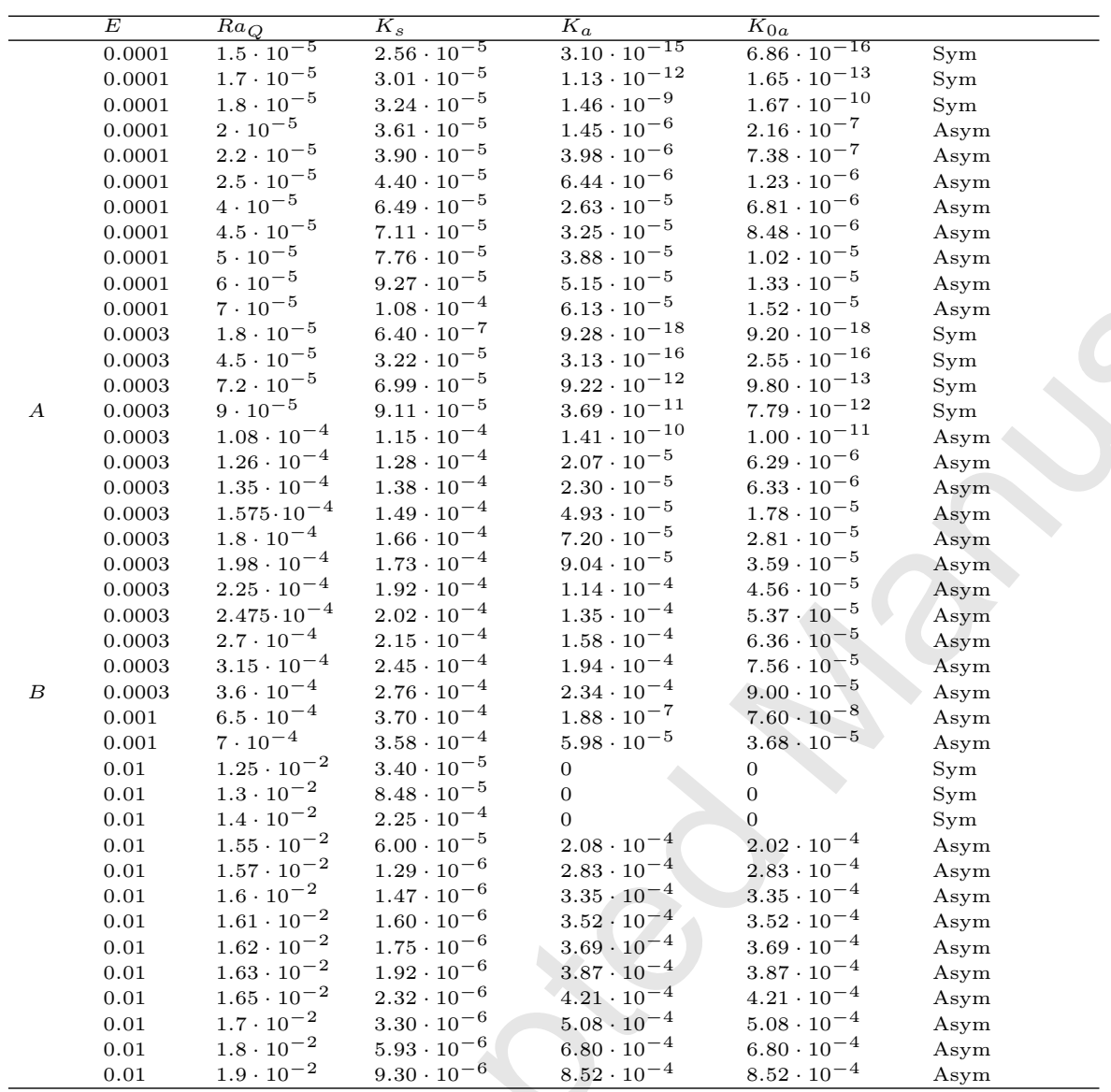

Table 1: Numerical models and results for hydrodynamic simulations. See text for the definitions of input parameters and output quantities. In all simulations we impose $\operatorname{Pr}=1$ and $P m=5$. The first column labels $A$ and $B$ tag runs which are specifically referred to in the text. The last column characterizes the resulting flow regime: 'Sym' and 'Asym' for simulations which are in a symmetric and asymmetric regime respectively (see section 3.2 for definitions). 


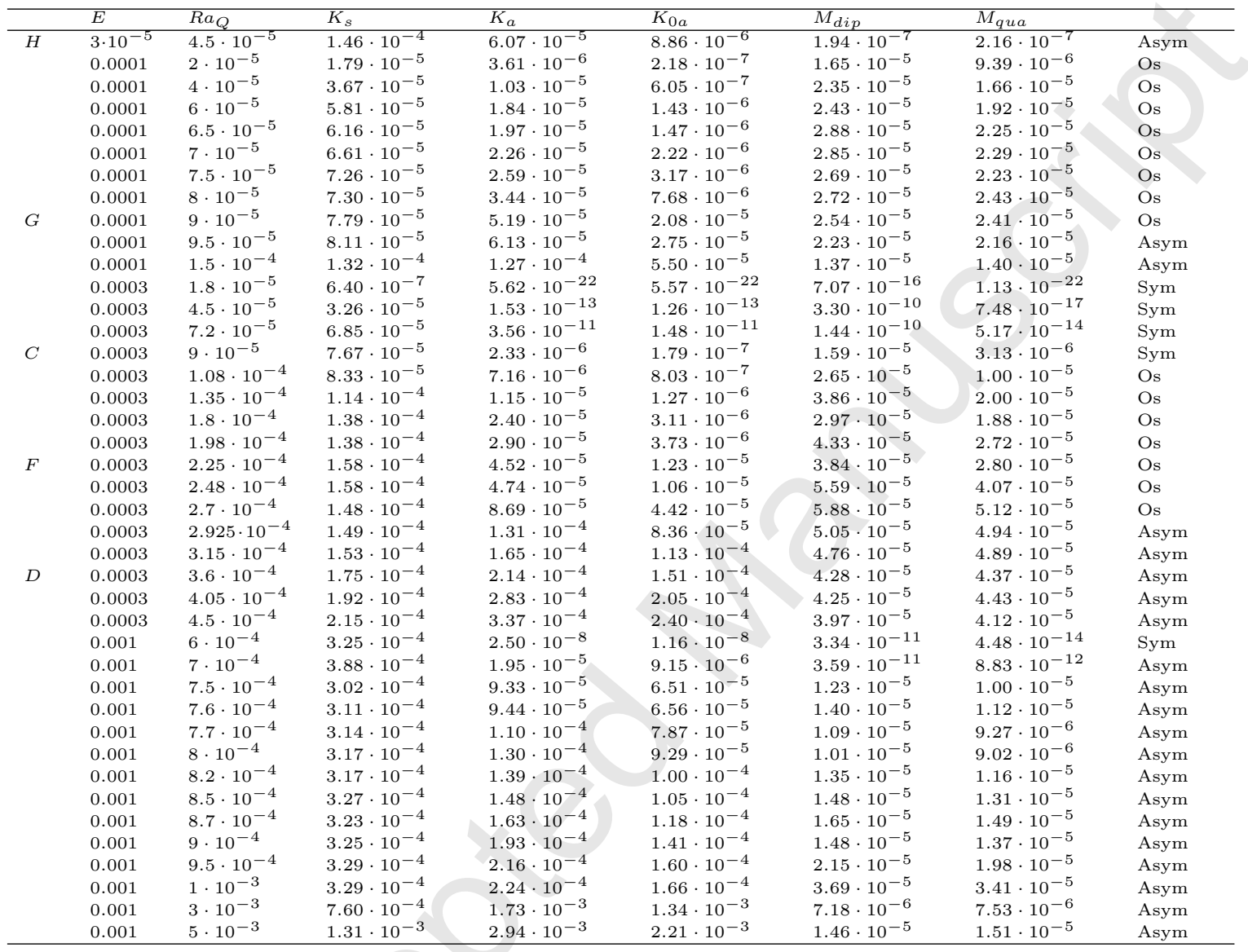

Table 2: Numerical models and results for dynamo simulations. See text for the definitions of input parameters and output quantities. In all simulations we impose $\operatorname{Pr}=1$ and $P m=5$, except in simulation $H$ in which $P m=1$. The first column labels $C$ to $H$ tag runs which are specifically referred to in the text. The last column characterizes the flow regime: 'Sym', 'Os' and 'Asym' for simulations which are in a symmetric, oscillating and asymmetric regime respectively (see section 3.2 and 4.1 for definitions). 
temperature profile outside the cylinder tangent to the inner core.

The time averaged kinetic energy density $K$ is defined as follows:

$$
K=\frac{1}{2 V_{S}}\left\langle\int_{V_{S}} \mathbf{u}^{2} d V\right\rangle
$$

where $V_{S}$ is the shell volume and the angled brackets indicate a time averaging operator. Using this template, we additionally define:

- the time averaged kinetic energy density contained in the equatorially antisymmetric, axisymmetric (EAA) flow component $K_{0 a}$,

- the time averaged kinetic energy density contained in equatorially antisymmetric modes $K_{a}$,

- the time averaged kinetic energy density contained in equatorially symmetric modes $K_{s}$.

In the present study, it is understood that an 'equatorially symmetric' vector field $\mathbf{u}$ is left unchanged by the operator $\Gamma$ which describes mirror-reflection through the equatorial plane, i.e. $\Gamma \mathbf{u}=\mathbf{u}$, while an 'equatorially antisymmetric' vector field is such that $\Gamma \mathbf{u}=-\mathbf{u}$.

We similarly define a time averaged magnetic energy density $M$ at the external boundary of the model:

$$
M=\frac{1}{2 S_{c m b}}\left\langle\int_{S_{c m b}} \mathbf{B}^{2} d S\right\rangle
$$

where $S_{c m b}$ is the surface of the sphere (at the CMB). Using this template, we also define:

- the time averaged CMB magnetic energy related to modes of dipole parity (odd $l+m$ in spherical harmonics) $M_{d i p}$,

- the time averaged CMB magnetic energy related to modes of quadrupole parity (even $l+m) M_{q u a}$.

Another output quantity $f_{\text {hem }}$ is used to characterize the hemisphericity of the magnetic field at the CMB:

$$
f_{\text {hem }}=\frac{\max \left[M^{S}, M^{N}\right]}{M},
$$




\begin{tabular}{rrr}
\hline$E$ & $R a_{Q c}$ & $m_{c}$ \\
\hline $10^{-6}$ & $1.08 \cdot 10^{-9}$ & 38 \\
$3 \cdot 10^{-6}$ & $6.80 \cdot 10^{-9}$ & 26 \\
$10^{-5}$ & $5.18 \cdot 10^{-8}$ & 17 \\
$3 \cdot 10^{-5}$ & $3.34 \cdot 10^{-7}$ & 12 \\
$5 \cdot 10^{-5}$ & $7.98 \cdot 10^{-7}$ & 10 \\
$10^{-4}$ & $2.61 \cdot 10^{-6}$ & 7 \\
$3 \cdot 10^{-4}$ & $1.72 \cdot 10^{-5}$ & 5 \\
\hline
\end{tabular}

Table 3: Critical Rayleigh number $R a_{Q c}$ and azimuthal wavenumber $m_{c}$ for the most linearly unstable equatorially symmetric convection mode.

where $M^{S}$ and $M^{N}$ are the time averaged magnetic energy densities contained in the Southern and Northern hemispheres. The hemisphericity factor $f_{\text {hem }}$ is equal to 0.5 for a purely dipolar field and has the value 1 for a purely hemispherical field.

\section{Results for convection without dynamo action}

In this section we introduce the results for secular cooling-driven convection in a rotating sphere without dynamo action. Starting from a non-convective stable state at low Rayleigh number, we introduce the main hydrodynamic transitions found when we progressively increase the forcing.

\subsection{Linear stability results: the onset of convection}

The first hydrodynamic transition corresponds to the onset of convection and occurs when the modified Rayleigh number reaches a first critical value $R a_{Q c}$. We start introducing the onset of convection in our system because it gives the framework for the nonlinear simulations presented in the following parts.

For each value of the azimuthal wavenumber $m$ and each value of the modified Rayleigh number, two growth-rates can be calculated using the linear version of the code PARODY: one for equatorially symmetric modes and one for equatorially antisymmetric modes. Indeed, these two families of modes are not coupled in the linearized equations.

We found that the first unstable modes are equatorially symmetric, nonaxisymmetric modes, as expected from previous theoretical studies (Busse, 1970; Jones et al., 2000). Table 3 lists the critical Rayleigh number and azimuthal wavenumber for each studied value of the Ekman number. Fig.2 shows that $R a_{Q c} / E^{5 / 3}$ converges towards an asymptote which is in good agreement with the 


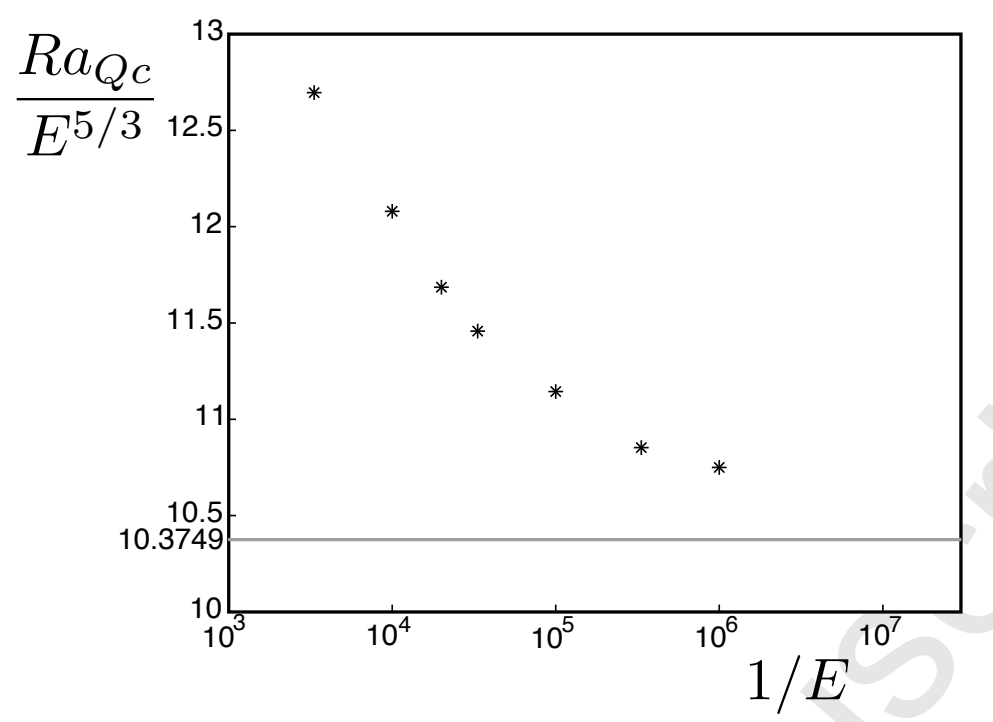

Figure 2: Convection onset. Stars: $R a_{Q c} / E^{5 / 3}$ versus $1 / E$ (logarithmic scale). The grey line is the asymptote predicted by the theory of Jones et al. (2000) with slightly different boundary conditions (see text).

value $10.3749(\approx 10.4)$ obtained by Jones et al. $(2000)$. It must be pointed out that Jones et al. (2000) used slightly different boundary conditions (fixed temperature and stress-free) at the external boundary, while we presently use a fixed flux condition for geophysical relevance and we consider rigid boundaries. However, as the temperature gradient in the bulk of the fluid is the same in our and their study, we do not expect the asymptote to be shifted by a dramatic amount, as confirmed by our numerical results. The asymptotic behavior of the critical modified Rayleigh number in the limit $E \rightarrow 0$ is thus approximated by:

$$
R a_{Q c} \approx 10.4 \cdot E^{5 / 3}
$$

In terms of critical canonical Rayleigh number $R a_{c}$, this corresponds to the following asymptotic behavior: $R a_{c} \approx 10.4 \cdot E^{-4 / 3}$. The exponent value $-4 / 3$ for the Ekman number dependence of the critical Rayleigh number is a robust feature of the onset of convection in rotating spheres or shells: it is expected from analytical consideration (Busse, 1970; Jones et al., 2000) and has subsequently been found in numerical studies (Dormy et al., 2004) for other geometries and boundary conditions.

As illustrated in Fig.3, the velocity structures at onset correspond to quasigeostrophic Rossby waves that vary slowly in $z$-direction. These waves form a set of non-axisymmetric vortices aligned with the rotation axis as predicted by Busse 

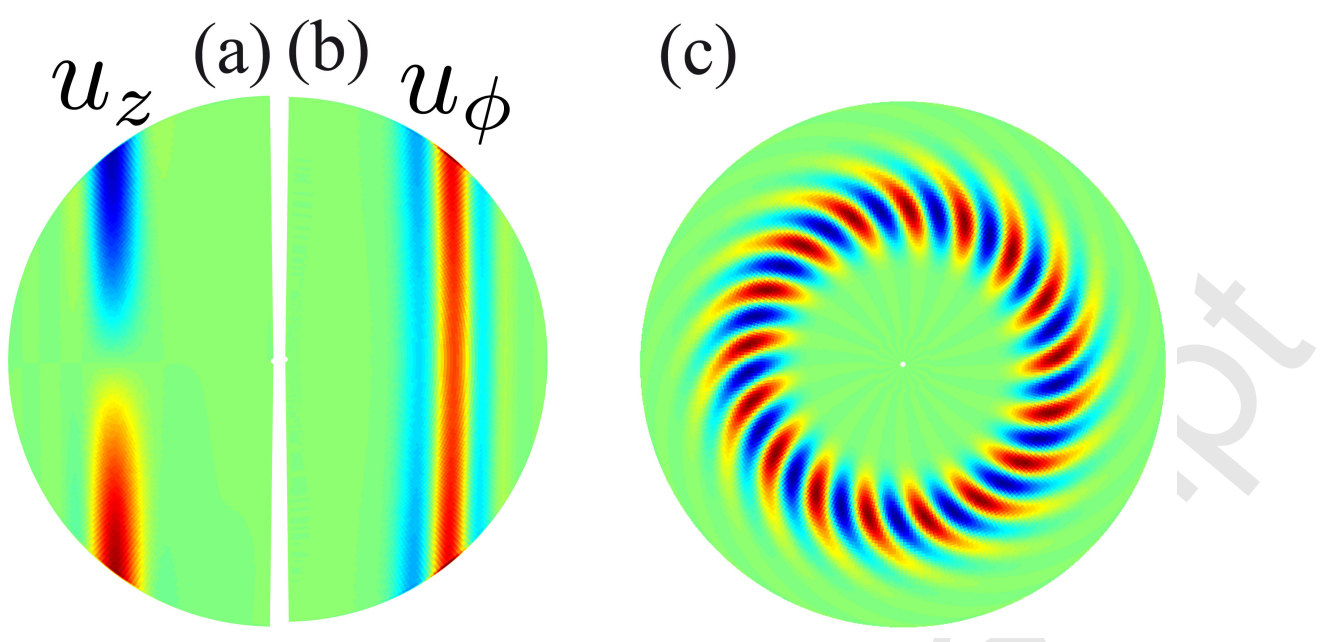

Figure 3: Velocity structures at onset for $E=10^{-5}$ and $\operatorname{Pr}=1$. (a), Meridional section of the $z$-component of velocity. (b), Meridional section of the azimuthal velocity field. (c), Equatorial section $(\theta=\pi / 2)$ of the $z$-component of vorticity.

(1970). The azimuthal wavenumber of the first unstable modes $m_{c}$, is expected to vary such that $m_{c} \propto E^{-1 / 3}$ (Busse, 1970; Jones et al., 2000). The values we found for $m_{c}$ are reported in Table 3 and are in agreement with the expected trend.

A second important family of convective modes is the axisymmetric family. At first sight it can seem of secondary importance to study the linear stability of this family into detail since we previously saw that the first unstable modes are nonaxisymmetric at high rotation rates (Geiger and Busse (1981) have shown that axisymmetric modes can be preferred at low rotation rates). However, as announced in section 1 and developed in the following section 3.2, the axisymmetric modes acquire a crucial importance in our nonlinear simulations. We thus compute (Table 4) the linear threshold of instability for the axisymmetric modes $R a_{Q a 0}$. Indeed, these results will be required in section 3.2 in order to determine if the emergence of EAA modes in nonlinear simulations is related to their linear instability. Within a margin of error of $20 \%$ (which corresponds to the misfit between the results of Roberts (1965) and Bisshopp and Niiler (1965)), our numerical results are compatible with both the asymptotes found by Roberts (1965), which yields:

$$
R a_{Q a 0} \approx 52.2 \cdot E^{5 / 3},
$$

and Bisshopp and Niiler (1965), which yields:

$$
R a_{Q a 0} \approx 61.3 \cdot E^{5 / 3},
$$


although the thermal boundary conditions are different and a small inner sphere is present in our study. Unlike the non-axisymmetric modes, the most linearly unstable axisymmetric mode belongs to the equatorially antisymmetric family. Its pattern (Fig.4) corresponds to a single convection cell carrying heat away in the direction of the rotation axis, whereas the first unstable non-axisymmetric modes convect heat in the cylindrical radial direction. As the axial circulation gets close to the upper and lower boundaries, the flow is diverted and couples with the Coriolis force to give rise to an equatorially antisymmetric, zonal circulation. As in the case of non-axisymmetric convection (Busse, 1970), viscous forces on short length scales of order $E^{1 / 3}$ are required to overcome the two-dimensional constraint of the Taylor-Proudman theorem. Then, the thickness of the axial cell is of order $E^{1 / 3}$ (Roberts, 1965) and motion in the cell is quasi-geostrophic, slowly varying in $z$-direction.

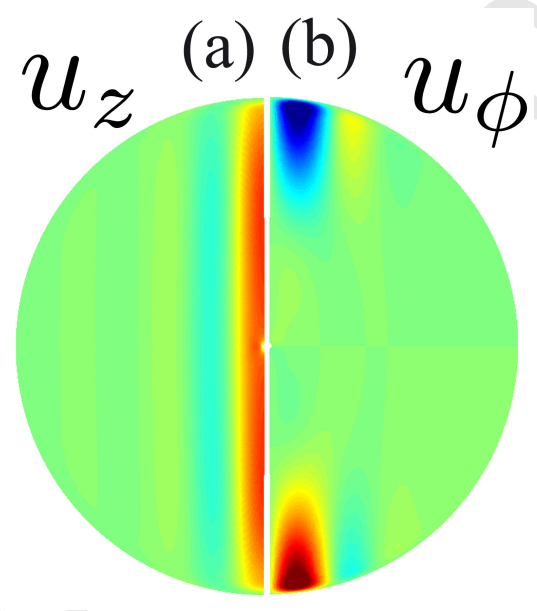

Figure 4: First unstable axisymmetric convection mode at $E=3 \cdot 10^{-4}$ and $\operatorname{Pr}=1$. (a), Meridional section of the $z$-component of velocity. (b), Meridional section of the azimuthal velocity field.

In summary, the linear stability analysis performed in the case of rotating convection driven by secular cooling confirms the theoretical results obtained with slightly different boundary conditions: equatorially symmetric, non axisymmetric vortices are the most linearly unstable modes, and the first linearly unstable axisymmetric modes are equatorially antisymmetric. The critical canonical Rayleigh numbers for both families vary as $E^{-4 / 3}$ when $E \rightarrow 0$. Planetary core dynamos are located largely above the onset of convection and nonlinear simulations are required to go further. 


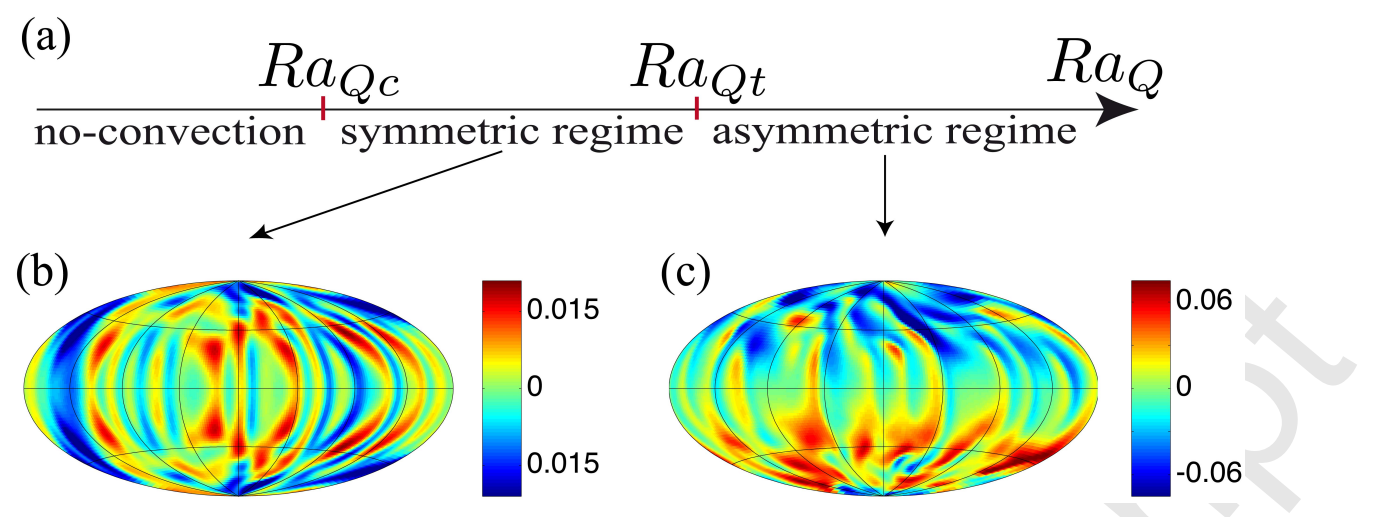

Figure 5: (a), Schematic representation of the two main hydrodynamic transitions found when increasing the modified Rayleigh number: from a non-convective state to the classical symmetric regime at $R a_{Q c}$ (onset of convection) and then, at $R a_{Q t}$, from the symmetric regime to the asymmetric regime (characterized by the emergence of an EAA mode). (b)-(c), Snapshots of azimuthal velocity field at radius $r=0.88$ (Hammer projection), hydrodynamic simulations. (b), Simulation $A$. (c), Simulation $B$ (parameters reported in Table 1).

\begin{tabular}{rrr}
\hline$E$ & $R a_{Q a 0}$ & $R a_{Q t}$ \\
\hline $10^{-4}$ & $8.37 \cdot 10^{-6}$ & $1.95 \cdot 10^{-5}$ \\
$3 \cdot 10^{-4}$ & $5.00 \cdot 10^{-5}$ & $1.07 \cdot 10^{-4}$ \\
$10^{-3}$ & $3.34 \cdot 10^{-4}$ & $6.28 \cdot 10^{-4}$ \\
$10^{-2}$ & $1.41 \cdot 10^{-2}$ & $1.41 \cdot 10^{-2}$ \\
\hline
\end{tabular}

Table 4: Critical Rayleigh numbers $R a_{Q a 0}$ for the linear onset of axisymmetric convection (EAA mode), and $R a_{Q t}$ for the nonlinear emergence of the EAA mode (see section 3.2).

\subsection{Nonlinear simulation results: transition towards the asymmetric regime}

When we increase the Rayleigh number slightly above onset, we found that non-axisymmetric vortices aligned with the rotation axis (equatorially symmetric structures) remain the main convective features, even though the flow becomes chaotic and small-scale structures appear. This result can be seen in Fig.5(b) which shows results obtained with simulation $A$ (with $R a_{Q} \approx 5 R a_{Q c}$, see Table 1 ). The columnar structures tend to satisfy the Taylor-Proudman theorem and the flow is said to be in a symmetric regime as indicated in Fig.5(a) which gives a schematic representation of the main hydrodynamic transitions found when increasing the modified Rayleigh number. Most of the previously studied nonlinear numerical simulations are located in this symmetric regime (see for instance Olson et al., 1999).

By further increasing the forcing, we found that the flow undertakes an un- 


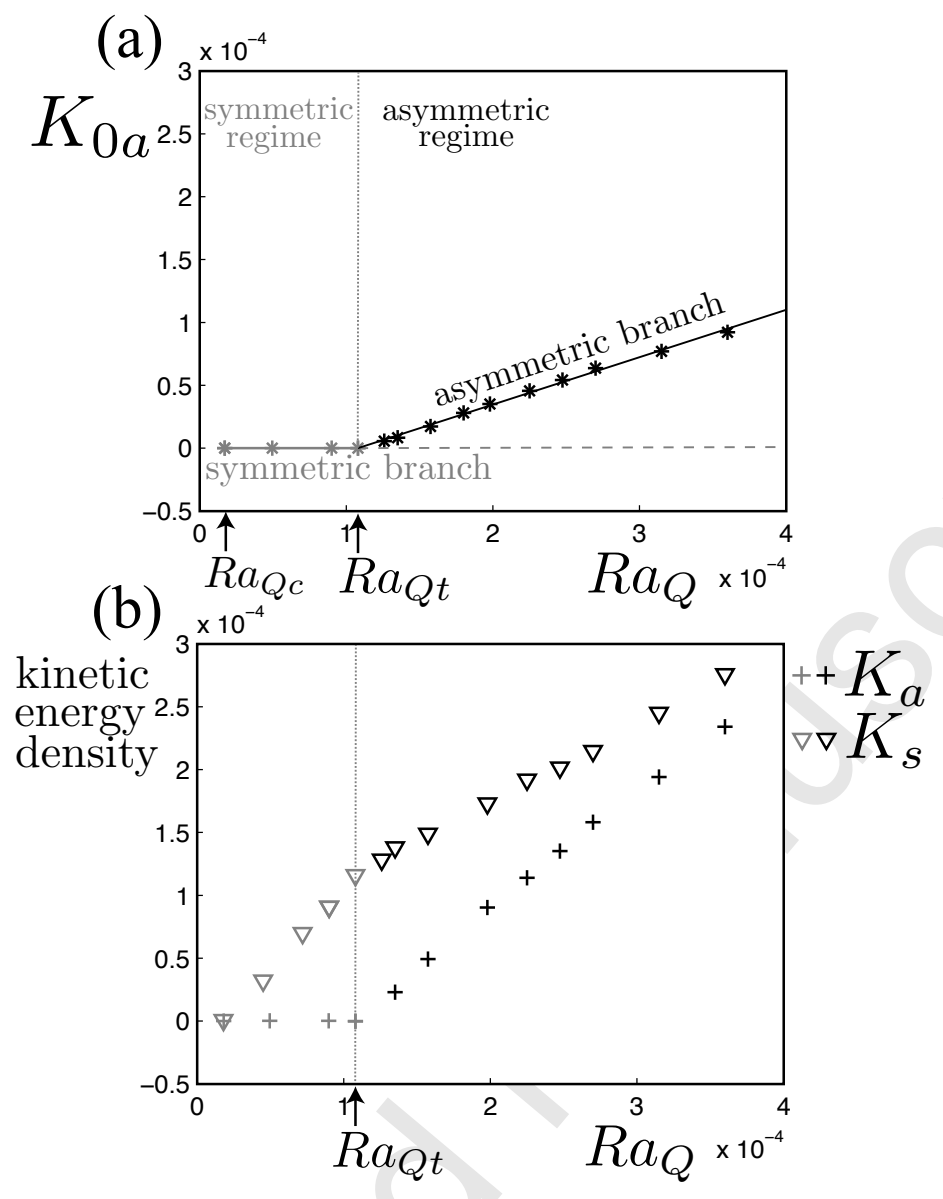

Figure 6: Bifurcation diagram showing $K_{0 a}$ (stars), $K_{a}$ (crosses) and $K_{s}$ (triangles) versus $R a_{Q}$ at $E=3 \cdot 10^{-4}$. Solution branches are identified in (a) since $K_{0 a}$ is the energy contained in the mode which emerges at the transition of interest. Solid and dashed curves refer to linearly stable and unstable solutions respectively. $R a_{Q t}$ locates the emergence of the asymmetric solution branch. To estimate the value of $R a_{Q t}$ we look for $R a_{Q t}$ and the constant $a$ such that $K_{0 a}$ is best scaled (in the sense of the least squares) by $a\left(R a_{Q}-R a_{Q t}\right)$ on the asymmetric branch.

expected transition when the modified Rayleigh number reaches a second critical value $R a_{Q t}$ (values reported in Table 4). Fig.5(a) shows a schematic representation of this transition and Fig. 6 serves as a bifurcation diagram. At the onset of convection $\left(R a_{Q c} \approx 0.17 \cdot 10^{-4}\right)$, the symmetric solution branch $\left(K_{0 a}<<K_{s}\right)$ emerges. At $R a_{Q t} \approx 1.07 \cdot 10^{-4}$, the symmetric branch looses stability and a new branch of solutions, which is characterized by a rapid increase of $K_{0 a}$, emerges through a supercritical pitchfork bifurcation. This branch of solutions is called asymmetric branch because 
it characterizes equatorially asymmetric solutions in which the EAA kinetic energy density $K_{0 a}$, and the equatorially symmetric kinetic energy density $K_{s}$, become of the same order of magnitude (Fig. 6). The asymmetric regime is unexpected since the amplitude of equatorially antisymmetric modes has always been found to be much smaller than the amplitude of equatorially symmetric modes in previous numerical simulations (Olson et al., 1999; Christensen and Aubert, 2006; Sakuraba and Roberts, 2009). The EAA mode is the dominant equatorially antisymmetric mode since almost half of $K_{a}$ is contained in this mode $\left(K_{0 a} \approx 0.44 K_{a}\right)$. Equatorially antisymmetric, non-axisymmetric modes also emerge at $R a_{Q t}$, with an energy density equal to $K_{a}-K_{0 a}$. However, we find that these modes do not emerge spontaneously, contrary to the EAA mode, but result from nonlinear interactions between the EAA mode and equatorially symmetric modes. The spatial structure of these modes is indeed strongly correlated with that of equatorially symmetric, non-axisymmetric modes. Thus, in the asymmetric regime, the dominant (and dynamically important) structures correspond to a superposition of columnar, equatorially symmetric modes and an EAA mode (Fig. 5(c)).

We found that, at low Ekman numbers $\left(E \leq 10^{-3}\right), R a_{Q t}$ is located above the linear threshold of instability of EAA modes $R a_{Q a 0}$ (Table 4). This result means that the emergence of an EAA mode in our nonlinear simulations can not be explained by linear stability analysis if $E \leq 10^{-3}$. Thus, the asymmetric branch emerges from the equatorially symmetric, columnar convection which has to be seen as the new basic state. We checked numerically that $R a_{Q t}$ corresponds indeed to the threshold of linear instability of EAA modes with respect to a purely equatorially symmetric basic state. The bifurcation at $E=10^{-2}$ is a very isolated case since $R a_{Q t}=R a_{Q a 0}$ (Table 4 ). In this case the bifurcation can be described in terms of interactions between two linearly unstable modes: an equatorially symmetric mode of order $m=1$ and an EAA mode. Since we are looking for asymptotic behaviors in the limit $E \rightarrow 0$, we will not consider the slowly rotating cases $E \geq 10^{-2}$ for the determination of the regime boundaries.

Fig.7 gives a schematic view of the EAA mode which emerges in the asymmetric regime: the azimuthal velocity field is organized into two large equatorially antisymmetric vortices, one in each hemisphere. Contrary to the two-cell meridional circulation of the symmetric regime (Olson et al., 1999), the time-averaged meridional circulation induced by the EAA mode is organized in only one cell. The fluid goes from one pole to the other passing through the center of the sphere. 


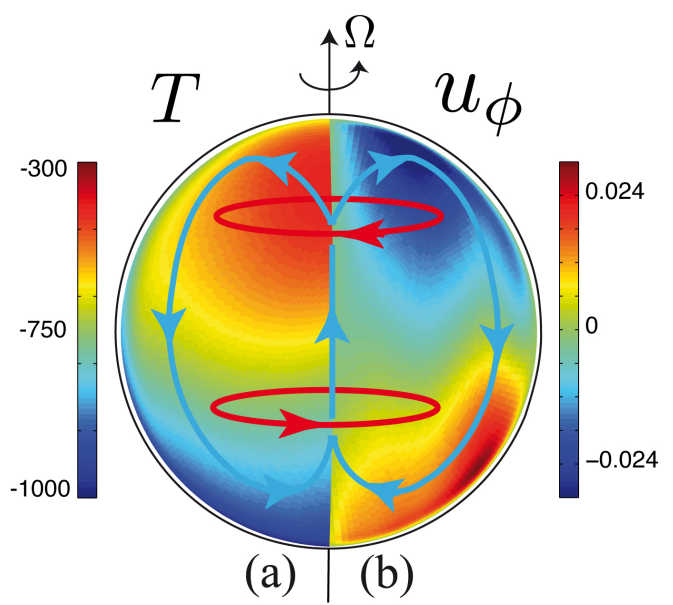

Figure 7: Arrows: schematic representation of the time-averaged EAA mode (azimuthal and meridional flows) which emerges in the asymmetric regime. (a), Meridional section (arbitrary azimuth) of the time-averaged temperature field in asymmetric simulation $B$ (parameters reported in Table 1). (b), Same as (a) for the time-averaged azimuthal velocity field.

As a consequence of this equatorially antisymmetric meridional circulation, the temperature profile has a considerable equatorially antisymmetric component (Fig.7(a)).

The dynamics of the asymmetric regime is strongly influenced by rotation since the local Rossby number (Christensen and Aubert, 2006) remains inferior to 0.08 in all our asymmetric simulations. We find that the equatorially asymmetric azimuthal velocity field results from meridional variation of the asymmetric temperature field through a thermal wind mechanism, which is characterized by a balance between the Coriolis, pressure gradient and buoyancy forces. Taking the $\phi$-component of the curl of the momentum equation, and retaining only the above forces, we have:

$$
\frac{\partial u_{\phi}}{\partial z}=\frac{R a_{Q}}{2 r_{0}} \frac{\partial T}{\partial \theta}
$$

Fig. 8 shows a high degree of similarity between the right-hand side and left-hand side terms of equation (16), thus confirming that equation (16) captures the flow dynamics inside the shell (except near the boundaries where the viscous term in equation (1) is not negligible). The term $\partial T / \partial \theta$ is globally negative in the whole shell as a consequence of the equatorially antisymmetric component of the temperature profile shown in Fig.7(a). Then, according to equation (16), $\partial u_{\phi} / \partial z$ is also negative, and this is coherent with an antisymmetric azimuthal flow organized in 


$$
<\frac{\partial u_{\phi}}{\partial z}>_{\phi}=<\frac{R a_{Q}}{2 r_{0}} \frac{\partial T}{\partial \theta}>_{\phi}
$$

(a)

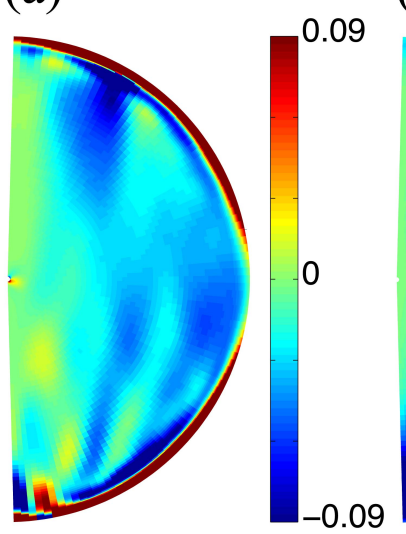

(b)

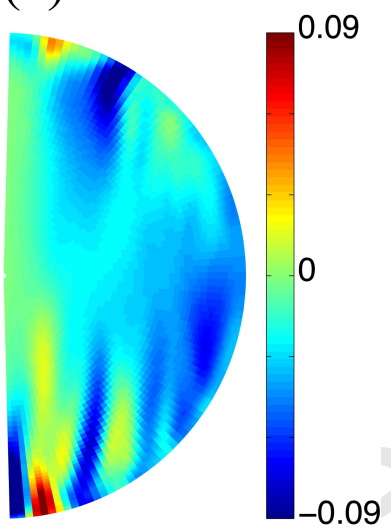

Figure 8: Comparison between (a) a snapshot of the $\phi$-average of the left-hand side term of equation (16), and (b) a snapshot of the $\phi$-average of the right-hand side term of the same equation. Results obtained using asymmetric simulation $B$ (parameters indicated in Table 1).

two vortices as we find in our asymmetric simulations (Fig.7(b)).

The time-averaged zonal velocity field is also in equilibrium with the timeaveraged convective axial velocity field. In our nonlinear simulations, we have found that this equilibrium arises through Ekman pumping. In the Southern hemisphere in Fig.7, the fluid is rotating faster than the external boundary, inducing a meridional flow that converges towards the center of the vortex. Conversely, the time-averaged meridional flow diverges from the center of the vortex in the Northern hemisphere. The axial velocity $v_{z}$ is then related to the vertical vorticity $\omega_{z}$ by $v_{z}=O\left(E^{1 / 2} \omega_{z}\right)$. To check this hypothesis we computed the ratio

$$
r_{E}=\frac{\max \left|\left\langle\left\langle v_{z}\right\rangle\right\rangle_{\phi}\right|}{E^{1 / 2} \max \left|\left\langle\left\langle\omega_{z}\right\rangle\right\rangle_{\phi}\right|}
$$

where \langle\rangle$_{\phi}$ and \langle\rangle denote the azimuthal and time averaging operators. Considering only the equatorially antisymmetric part of the velocity and vorticity fields, we find a mean value $\bar{r}_{E}=3.52$ and a standard deviation 1.6, meaning that this ratio remains of order 1, as expected in the case of an Ekman pumping mechanism, even though our configuration is far from being the ideal case of a unique rotating plate for which the classical Ekman pumping formula is derived.

The equations (1-2) and (5) and the boundary conditions have equatorial re- 
flection symmetry. Consequently, if $A(t)$ is the amplitude of the EAA mode $\mathbf{u}_{a}$, then $A \mathbf{u}_{a}$ and $-A \mathbf{u}_{a}$ are two dynamically equivalent solutions. This means that the solution for the EAA mode which is represented in Fig.7 is dynamically equivalent to the solution which can be obtained by reversing the arrows in Fig.7. In our simulations we indeed found both solutions. The system chooses one of the two and does not reverse towards the other. Thus, the EAA mode should emerge through a pitchfork bifurcation. As it would be in a canonical supercritical pitchfork bifurcation, $K_{0 a}$ is proportional to $\left(R a_{Q}-R a_{Q t}\right)$ in our numerical simulations (Fig.6(a)).

Considering the possible relationship between the emergence of a strong EAA mode and the smallness (or absence) of the inner core, we found the same hydrodynamic transition towards the asymmetric regime in a shell with aspect ratio $r_{i} / r_{o}=0.35$, provided the driving mode is the same (secular cooling with zero heat flux at the inner core). The critical value $R a_{Q t}$ is larger when $r_{i} / r_{o}=0.35$ than when $r_{i} / r_{o}=0.01$ (results not reported here) but the transition occurs at about the same static temperature difference in both cases. However, no transition to the EAA state has been found when a non-zero homogeneous heat flux or fixed temperature was imposed at the inner core boundary, suggesting that the presence of a thermal boundary layer with a positive incoming heat flux at the inner core boundary prevents the EAA mode from emerging. We presume that the EAA hydrodynamic transition is favored in our numerical simulations because the buoyancy driving allows for EAA convection carrying heat away in the direction perpendicular to the equatorial plane.

The different transitions found are represented in a $\left(1 / E, R a_{Q}\right)$ parameter space (Fig. 9). The transition between the symmetric and asymmetric regimes occurs at $R a_{Q t}$, which is best scaled (in the sense of the least squares) by:

$$
R a_{Q t} \approx 21.2 \cdot E^{1.51}
$$

\section{Results for convective dynamos}

We now turn to the study of the EAA mode in the presence of dynamo action. We first introduce the different hydrodynamic transitions found when allowing dynamo action and compare them with the transitions found in hydrodynamic simulations (section 3). Then we present the changes in magnetic field generation which are related to these hydrodynamic transitions. 


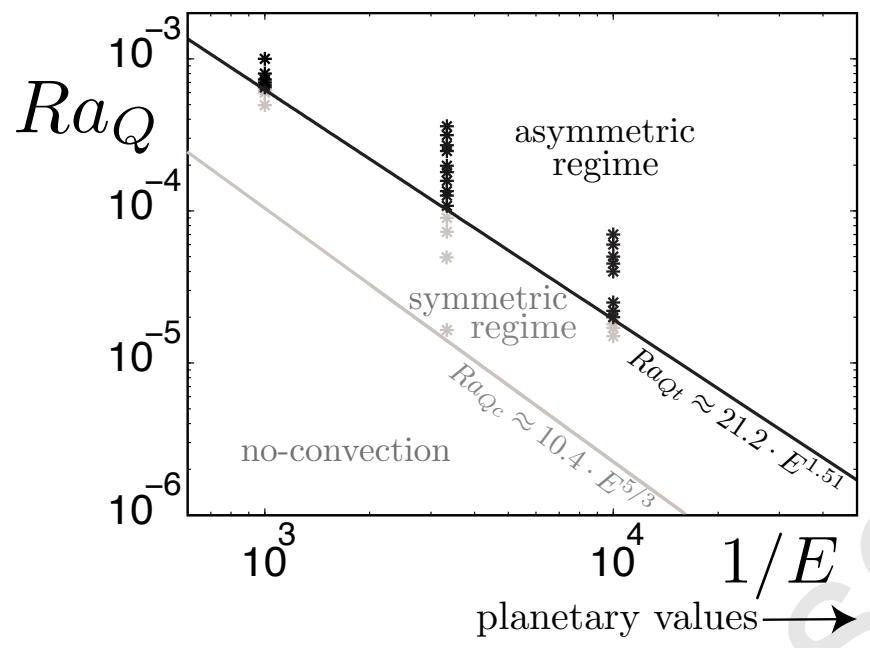

Figure 9: Phase diagram of the two main hydrodynamic transitions in the absence of dynamo action: from a non-convective state to the symmetric regime (light grey curve corresponds to the asymptotic behavior of $R a_{Q c}$ at low Ekman numbers according to equation (13)) and from the symmetric regime to the asymmetric regime (black curve). Light grey symbols: symmetric simulations. Black symbols: asymmetric simulations.

(a)
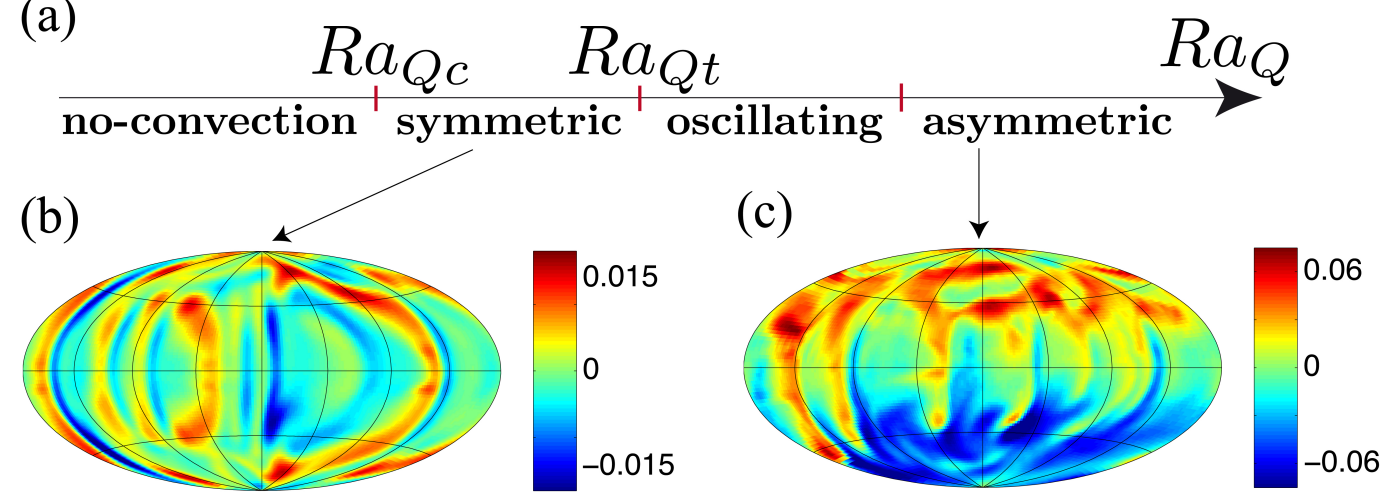

Figure 10: (a), Schematic representation of the main hydrodynamic transitions found when increasing the modified Rayleigh number and allowing dynamo action: from a non-convective state to the classical symmetric regime at $R a_{Q c}$ (onset of convection) and then, from the symmetric regime to the oscillating regime at $R a_{Q t}$ and finally from the oscillating regime to the asymmetric regime. (b)-(c), Snapshots of azimuthal velocity field at radius $r=0.88$ (Hammer projections). (b), Simulation $C$. (c), Simulation $D$ (parameters reported in Table 2).

\subsection{Hydrodynamic transitions}

Fig.10(a) gives a schematic representation of the different hydrodynamic transitions found when increasing the modified Rayleigh number and allowing dynamo 
action. The results for the linear onset of convection at $R a_{Q c}$ are identical to what we found in section 3.1 (without dynamo action) since the Lorentz force (third term in the right-hand side of equation (1)) is a nonlinear term. Increasing the modified Rayleigh number above onset we found a symmetric regime dominated by columnar, equatorially symmetric vortices as illustrated in Fig.10(b), similarly to the non-magnetic case.

By further increasing the forcing, the flow undertakes successive changes of regime which can be identified in the bifurcation diagram of Fig.11(a). When $R a_{Q}$ reaches the value $R a_{Q t}$ (previously computed in section 3.2), the symmetric solution branch $\left(K_{0 a}<<K_{s}\right)$ becomes unstable and the instantaneous value of $K_{0 a}$ starts oscillating in a chaotic manner between low values much smaller than $K_{s}$ (symmetric regime), and larger values of order $K_{s}$ (asymmetric regime). The flow is said to be in an oscillating regime, illustrated in Fig.12. Finally, when the forcing is strong enough $\left(R a_{Q} \approx 3 \cdot 10^{-4}\right)$, the flow reaches the asymmetric regime: the instantaneous value of $K_{0 a}$ remains large and does not reach the symmetric solution branch anymore. Similarly to the hydrodynamic case, the dominant (and dynamically important) modes in the asymmetric regime are the columnar, equatorially symmetric modes and the EAA mode (Fig.10(c)).

We found a similar bifurcation diagram (with a symmetric, oscillating and asymmetric regime) at $E=10^{-4}$. However we did not find any oscillating simulations at $E \geq 10^{-3}$ because the dynamo onset has not been overcome when $R a_{Q}$ reaches $R a_{Q t}$ at such Ekman numbers. Therefore, the bifurcation diagrams are similar to the ones obtained in hydrodynamic simulations if $E \geq 10^{-3}$. Since we are looking for asymptotic behaviors in the limit $E \rightarrow 0$, we will not consider cases in which $E \geq 10^{-3}$ for the determination of the regime boundaries.

The appearance of the oscillating regime when allowing dynamo action can be seen as a consequence of Ferraro's law of corotation (Ferraro, 1937): the axisymmetric magnetic field lines tend to follow the isocontours of $\left\langle u_{\phi} / s\right\rangle_{\phi}$ where $s$ is the cylindrical radius. At the beginning of an oscillation towards the asymmetric regime, the EAA flow component emerges because it is linearly unstable with respect to the symmetric regime (because $R a_{Q} \geq R a_{Q t}$ ). Then, the EAA mode distorts the isocontours of $\left\langle u_{\phi} / s\right\rangle_{\phi}$ which no longer follow the magnetic field lines. Consequently, an axisymmetric azimuthal magnetic field is created from stretching of the axisymmetric poloidal magnetic field by the EAA azimuthal 


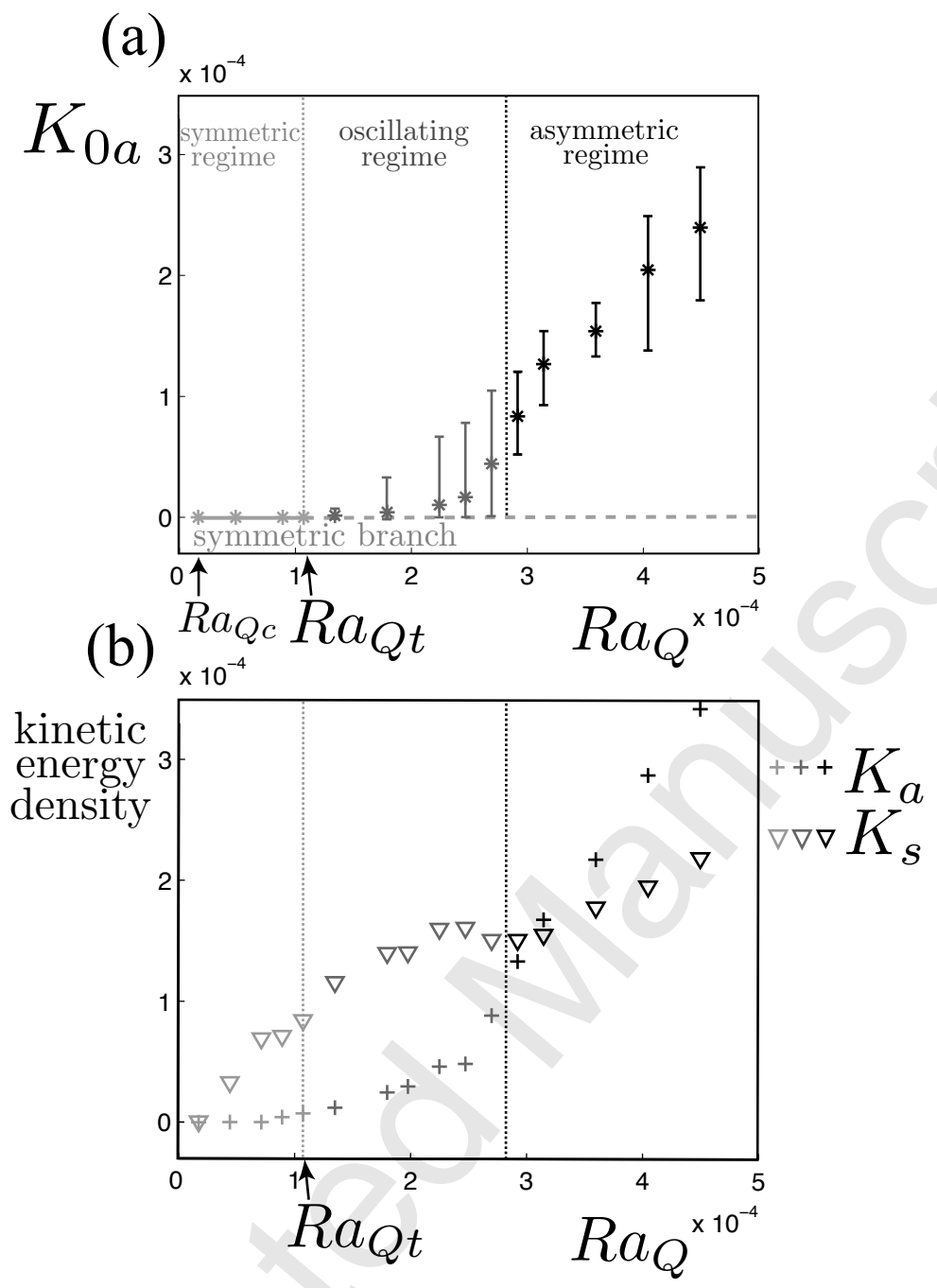

Figure 11: Bifurcation diagram at $E=3 \cdot 10^{-4}$ (when allowing dynamo action) showing $K_{0 a}$ (stars), $K_{a}$ (crosses) and $K_{s}$ (triangles) versus $R a_{Q}$. Vertical bars in (a) show the range of values taken by the instantaneous values of $K_{0 a}$. $R a_{Q t}$ corresponds to the emergence of the asymmetric branch introduced in the hydrodynamic study (computed in section 3.2). Light grey, medium grey and black symbols correspond to symmetric, oscillating and asymmetric simulations respectively (see text). Note that $K_{0 a}$ is not exactly equal to zero in the symmetric regime but very small compared to the scale of the figure.

flow through an $\omega$-effect, which increases the magnetic tension along the meridional field lines. In agreement with Lenz law, the resulting Lorentz force tends to oppose the motion that increases the magnetic tension, i.e. reduces the EAA flow component. If the Lorentz force becomes strong enough, the flow returns its sym- 
(a)

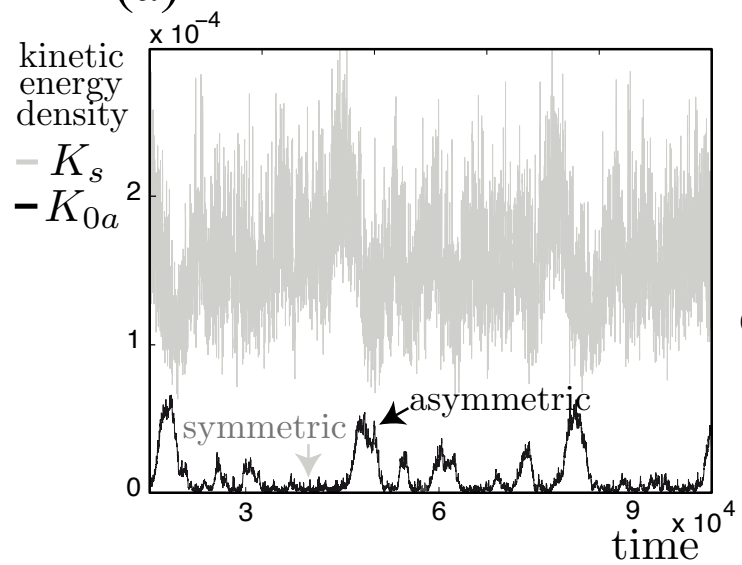

(b)

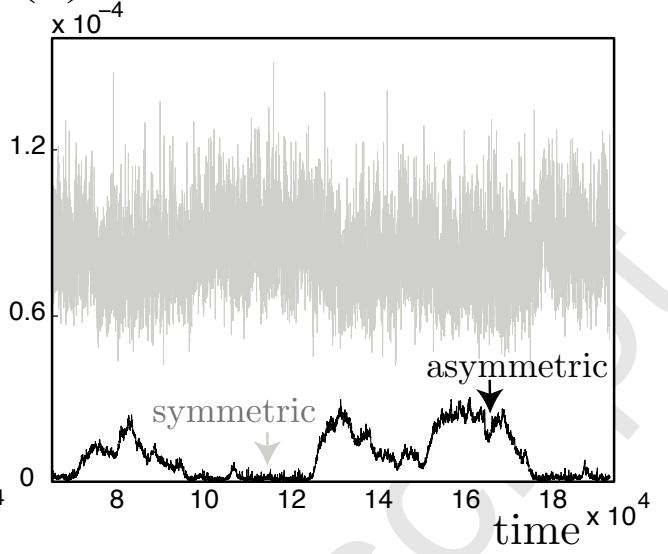

Figure 12: Instantaneous values for $K_{0 a}$ (black curve) and $K_{s}$ (light grey curve) versus time for oscillating simulations $F\left((\mathbf{a}), R a_{Q}\right.$ close to $\left.R a_{Q t}\right)$ and $G\left((\mathbf{b}), R a_{Q}\right.$ further away from $R a_{Q t}$ ) (Table 2).

metric regime. Thus, the closer we get to $R a_{Q t}$ in the oscillating regime, the smaller the growth-rate value of the EAA flow component becomes and the faster the Lorentz force will be able to restore the symmetric state. As a consequence, for Rayleigh numbers located just above $R a_{Q t}$, we observe rather bursts towards the asymmetric regime than oscillations (Fig.12(a)).

The EAA mode forms one axisymmetric vortex in each hemisphere, one cyclone and one anticyclone. The geometry of the time-averaged EAA mode in Fig.7 remains unchanged when dynamo action is present.

Similarly to Fig.9, Fig.13 summarizes the regime boundaries in a $\left(1 / E, R a_{Q}\right)$ parameter space when dynamo action is allowed. We emphasize here again that the boundary between symmetric and oscillating regimes is set by $R a_{Q}=R a_{Q t}$, where $R a_{Q t}$ is the forcing at which the transition from the symmetric to the asymmetric regime occurs in the hydrodynamic case. Its location is thus given by equation (18).

\subsection{Magnetic field structures: effects of the emergence of the EAA mode}

Figure 14 shows the qualitative effects of the transition from the symmetric to the asymmetric hydrodynamic regime on the dynamo-generated magnetic field. Fig.14(a) shows the results obtained with symmetric simulation $C$ (Table 2): the magnetic field is dipole dominated similarly to previously described numerical 


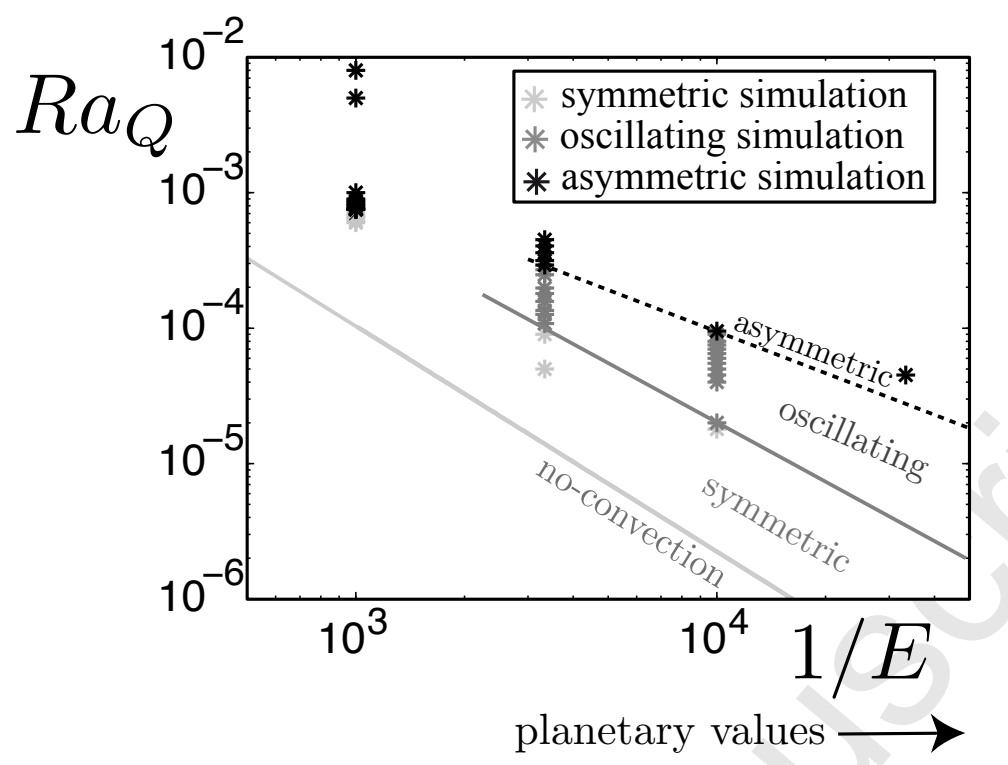

Figure 13: Phase diagram of the main hydrodynamic regimes when allowing dynamo action. Each symbol corresponds to one numerical simulation. Light grey, medium grey and black symbols correspond to symmetric, oscillating and asymmetric simulations respectively. The light grey curve corresponds to the asymptotic behavior of $R a_{Q c}$ given by equation (13). The medium grey curve corresponds to the best fit (in the sense of the least squares) for $R a_{Q t}$. The black dashed line corresponds to a tentative boundary regime between the oscillating and asymmetric regime.

dynamos. In contrast, in asymmetric simulation $D$ (Table 2), the magnetic field is hemispherical with high intensities in one hemisphere and weaker in the other (Fig.14(b)), not only at the CMB (top) but also at the surface of the planet (bottom). Thus, the hydrodynamic asymmetric regime can induce hemispherical dynamos.

The reason why the radial magnetic field becomes hemispherical in the asymmetric hydrodynamic regime can be qualitatively captured looking at the corresponding DMFI visualization (Aubert et al., 2008) (Fig.15). The surface magnetic flux is collected in the hemisphere where the EAA meridional flow converges. Near the pole, the converging EAA meridional flow is converted into flow downwellings. The ambient radial magnetic field is amplified by stretching within these downwellings, forming magnetic downwellings which are similar to the magnetic upwellings described in Aubert et al. (2008). In the other hemisphere, magnetic flux is dispersed by the divergent EAA flow and is thus much weaker. 

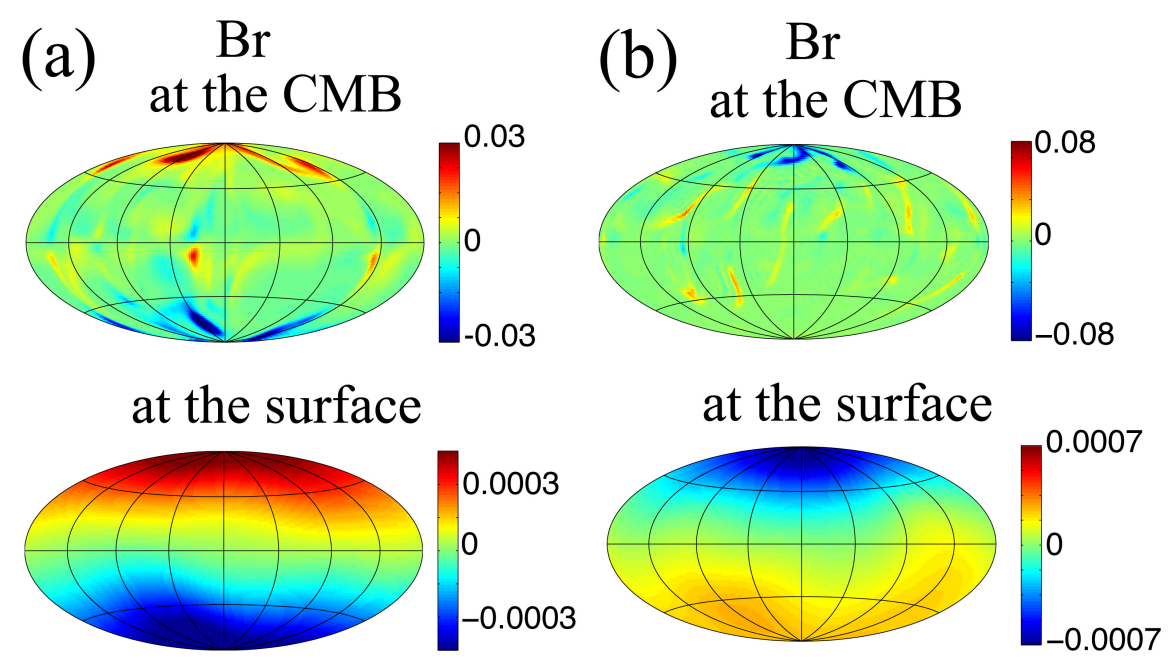

Figure 14: Snapshots of the radial magnetic field at the CMB (top) and at the surface of a Marslike planet (bottom) (Hammer projections). (a), Symmetric simulation C. (b), Asymmetric simulation $D$.

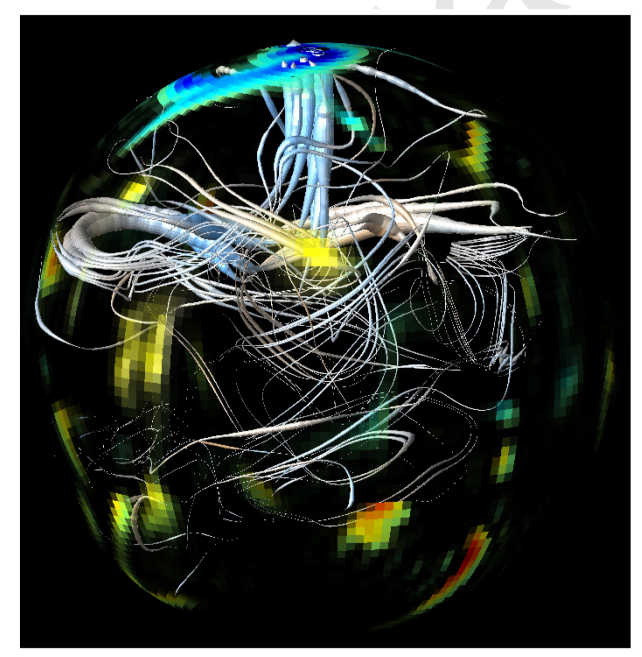

Figure 15: DMFI visualization of asymmetric simulation $D$ (Table 2). The outer boundary of the model is color-coded with the radial magnetic field. In addition, the outer boundary is made selectively transparent, with a transparency level that is inversely proportional to the local radial magnetic field. Field lines are displayed in grey, their thickness is proportional to $\mathbf{B}^{2}$ (for details see Aubert et al., 2008). 
(a)

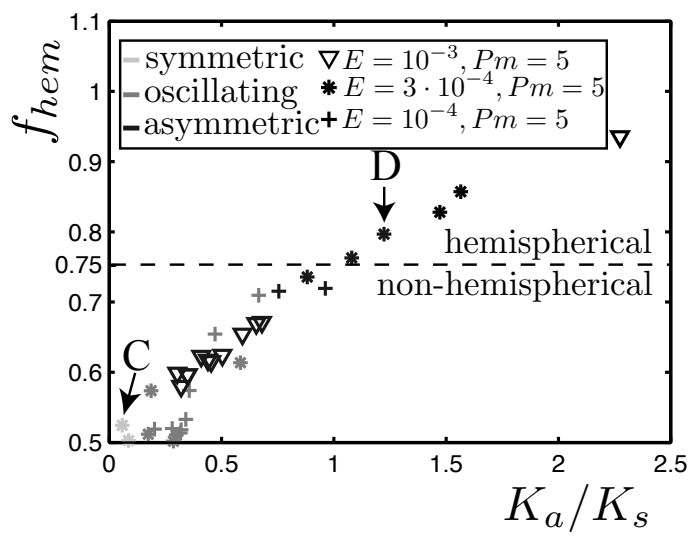

(b)

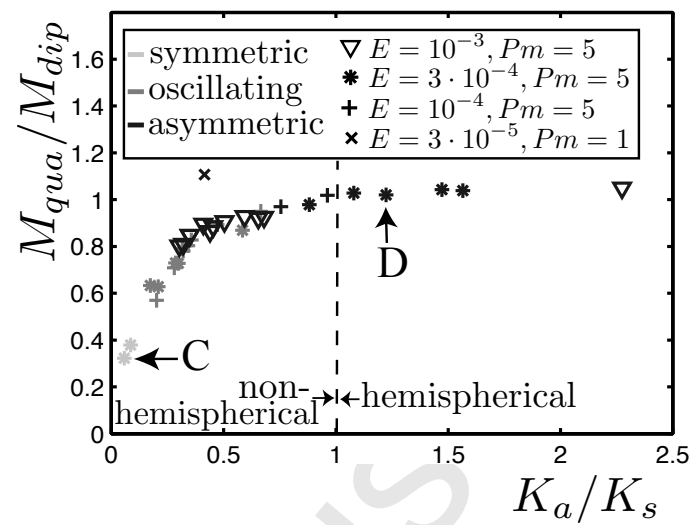

Figure 16: (a), Hemisphericity factor $f_{\text {hem }}$ versus $K_{a} / K_{s}$. (b), Magnetic energy parity ratio $M_{\text {qua }} / M_{\text {dip }}$ versus $K_{a} / K_{s}$. Light grey, medium grey and black symbols correspond to symmetric, oscillating and asymmetric simulations respectively. The dashed black line locates the transition from non-hemispherical to hemispherical dynamos at $f_{\text {hem }}=0.75$. The symbols $C$ and $D$ indicate the results obtained with simulations $C$ and $D$ respectively, which are illustrated in Fig.14.

In order to quantify this result, we computed the hemisphericity factor $f_{\text {hem }}$ (Fig.16(a)). A dynamo is said to be hemispherical if $f_{\text {hem }} \geq 0.75$ which means that one hemisphere contains at least $75 \%$ of the CMB magnetic energy. The ratio $K_{a} / K_{s}$, which measures the equatorial symmetry breaking of the flow, is a control parameter of the hemisphericity factor $f_{h e m}$, as shown by the univariate behavior in Fig.16(a). In symmetric simulations the flow is dominated by equatorially symmetric modes and $K_{a} / K_{s}$ has low values. In these symmetric simulations the hemisphericity factor is very close to 0.5 which means that these dynamos are not hemispherical, as illustrated with Fig.14(a). In asymmetric and oscillating simulations the ratio $K_{a} / K_{s}$ increases progressively from low values $(\sim 0.2)$ to large values $(\sim 2.3)$ due to the progressive emergence of the EAA mode. Fig.16(a) shows that the hemisphericity factor $f_{\text {hem }}$ increases almost linearly with $K_{a} / K_{s}$ and the transition from non-hemispherical to hemispherical dynamos is gradual. The hemisphericity factor reaches 0.75 when $K_{a} / K_{s} \approx 1($ at $P m=5)$. Several hemispherical dynamos $\left(f_{\text {hem }} \geq 0.75\right)$ are obtained, including the simulation of Fig.14(b). The reader may have expected the use of $K_{0 a} / K_{s}$ rather than $K_{a} / K_{s}$ in Fig.16(a) since the equatorial symmetry breaking of the flow is caused by the emergence of the EAA mode in our simulations. However, we find a less univariate behavior if we plot $f_{h e m}$ as a function 
of $K_{0 a} / K_{s}$ rather than $K_{a} / K_{s}$. This result suggests that equatorially antisymmetric, non-axisymmetric modes play a non-negligible role in the transition towards hemispherical dynamos. However, these nonaxisymmetric modes remain a consequence of the spontaneous emergence of the EAA mode.

Fig.16(b) shows that the equatorial symmetry breaking of the flow $K_{a} / K_{s}$, is also a control parameter of the magnetic field parity $M_{q u a} / M_{d i p}$ at fixed $P m$. Indeed, all the simulations are aligned on the same curve (with the exception of one simulation which has been obtained at a different value of $P m$ ). At fixed $P m, M_{q u a} / M_{d i p}$ increases when $K_{a} / K_{s}$ increases (due the emergence of the EAA mode in the oscillating and asymmetric regimes). When $K_{a} / K_{s}$ reaches $\sim 0.75$, $M_{q u a} / M_{d i p}$ saturates and remains close to 1: there is equipartition between magnetic energy contained in modes of dipole parity and magnetic energy contained in modes of quadrupole parity. We underline that several simulations have reached the equipartition of magnetic energy even though they are not hemispherical (for instance, multipole-dominated simulations). Note that we use $K_{a} / K_{s}$ rather than $K_{0 a} / K_{s}$ for the same reasons as in Fig.16(a).

\section{Discussion}

\subsection{Discussion of the numerical results}

At onset, convection driven by secular cooling (modeled by internal heating) in rapidly rotating spheres is very similar to what has been obtained for other geometries and boundary conditions: the first unstable modes are equatorially symmetric, non-axisymmetric vortices aligned with the rotation axis. By increasing the modified Rayleigh number above onset we found a flow regime which remains dominated by equatorially symmetric modes. These modes are in agreement with the Taylor-Proudman constraint. The flow is said to be in a symmetric regime and it is very similar to flows already described in previous numerical studies (Olson et al., 1999).

By further increasing the forcing, we found a transition towards a new flow regime, called the asymmetric regime. We have shown that the asymmetric regime is characterized by the emergence of an EAA mode (at $R a_{Q}=R a_{Q t}$ ), with an amplitude which becomes of the same order of magnitude as those of equatorially symmetric modes. This transition is unexpected. First, because the amplitude of equatorially antisymmetric modes has always been found to be much smaller than the amplitude of equatorially symmetric modes in previous studies (Olson et al., 1999; Christensen and Aubert, 2006; Sakuraba and Roberts, 2009). Second, because bifurcations are often 
related to symmetry breaking. Even though the emergence of the EAA mode breaks the equatorial symmetry, this mode has gained axisymmetry with respect to the columnar basic state on which it emerges. The occurrence of this transition highlights the need to study secondary instability mechanisms, especially for planetary systems which are far above the onset of primary instability.

The dynamics of the asymmetric regime is strongly influenced by rotation. The EAA mode comprises strong azimuthal thermal winds which induce two large-scale axial vortices: a cyclone in one hemisphere and an anticyclone in the other hemisphere. The related time-averaged meridional circulation is organized in only one cell. The EAA mode is the nonlinear manifestation of the first linearly unstable axisymmetric mode (considering a static basic state) studied by Roberts (1965) and Bisshopp and Niiler (1965). We underline that the EAA mode is an alternative way of carrying heat away while complying with the Taylor-Proudman constraint. As shown by equations (14) and (15), the critical modified Rayleigh number for axisymmetric convection is proportional to $E^{5 / 3}$, as is the critical Rayleigh number for non-axisymmetric convection (equation (13)). The Rayleigh number $R a_{Q t}$ for the nonlinear emergence of the EAA mode scales with the power 1.51 of the Ekman number (equation (18)), which is rather close to $5 / 3$.

For the EAA mode to emerge and become a dynamically meaningful mode, two conditions must be met: the buoyancy flux must vanish at the inner boundary and $R a_{Q}$ has to exceed $R a_{Q t}$. The reason why the asymmetric regime has not been previously observed stems from the fact that one of these two conditions was not met in earlier studies. The size of the inner core appears not to have effect on the transition towards the asymmetric regime. However, in a geophysical context, the presence of an inner core implies a non-zero buoyancy flux at the inner boundary. For that reason, the asymmetric regime is only expected in planetary systems that have not nucleated an inner core yet, and where convection is thus powered only by secular cooling (or radiogenic heating).

We have shown that the emergence of the EAA mode in the asymmetric hydrodynamic regime breaks the equatorial symmetry which controls the hemisphericity of the dynamo. Indeed, if the energy contained in the EAA mode is strong enough (i.e. the equatorial symmetry breaking of the flow $K_{a} / K_{s}$ is larger than $\sim 1$ ), then we obtain hemispherical dynamos in which at least $75 \%$ of the total magnetic energy at the CMB is contained in one hemisphere. The fact that an equatorial 
symmetry breaking of the flow can lead to hemispherical dynamos is a universal result related to fundamental symmetries in the governing equations, and can be captured using simple kinematic $\alpha^{2}$-dynamo models (Gallet and Petrelis, 2009). The equatorial symmetry breaking of the flow, due to the emergence of the EAA mode, leads to an equipartition between magnetic energy contained in modes of dipole parity and magnetic energy contained in modes of quadrupole parity in agreement with the low dimensional model proposed by Gallet and Petrelis (2009).

Hemispherical dynamos have been previously found in numerical simulations of convection and dynamo action in rotating shells (Grote and Busse, 2000; Simitev and Busse, 2005; Stanley et al., 2008). Fixed temperature and stress-free boundary conditions have been imposed in Grote and Busse (2000) and in Simitev and Busse (2005). Their hemispherical dynamos do not result from the same mechanism as ours. Indeed, we found that the antisymmetric kinetic energy remains at low values in their dynamo simulations $\left(K_{a} / K_{s} \approx 0.01\right.$ at $\operatorname{Pr}=1, \operatorname{Pm}=2$, $E=2 \cdot 10^{-4}$ and $\left.R a=6.5 \cdot 10^{5}\right)$ and it is exactly equal to zero in the corresponding hydrodynamic simulations. In Stanley et al. (2008), hemispherical dynamos result from the emergence of an EAA mode, as in our simulations, but this mode is forced by thermal boundary conditions in Stanley et al. (2008) while it spontaneously emerges in our study.

\subsection{Implications for the past martian dynamo}

The EAA mode of convection could be an attractive explanation for the asymmetry of Mars' crustal magnetic field without requiring any post-dynamo mechanism or any heat flux heterogeneity at the CMB. In the following we discuss first, whether the past martian dynamo could have been in an asymmetric hydrodynamic regime and, second, whether the asymmetric regime may generate hemispherical dynamos at Ekman numbers close to planetary values.

The past martian dynamo may have reached the asymmetric regime if $R a_{Q}$ was at least larger than $R a_{Q t}$ when the dynamo was active. One may use the scaling law (18) to estimate $R a_{Q t}$ in Mars' core: considering plausible parameter values given in Table 5 , we find that $E$ is roughly within the range $5 \cdot 10^{-15}-8 \cdot 10^{-15}$ in Mars' core and $R a_{Q t}$ within the range $5 \cdot 10^{-21}-10^{-20}$. The past martian CMB heat flux depends on the mechanism of heat transfer which is considered. Considering a stagnant lid mantle convection the maximum heat flux is expected to be about $60 \mathrm{~mW} \mathrm{~m}^{-2}$ (Nimmo and Stevenson, 2000; Breuer and Spohn, 2003; Stevenson et al., 1983) whereas if we consider an overturn after magma ocean crystallization it is about $600 \mathrm{~mW} \mathrm{~m}^{-2}$ (Elkins-Tanton et al., 2005). Plate tectonics has been suggested for Mars but is not coherent with little remixing of crust 
and mantle as indicated by geochemistry. In addition Breuer and Spohn (2003) have shown that it is difficult to reconcile crust production required by geological constraints and the presence of a core-dynamo using a model that includes plate tectonics. We note that, in the case of plate tectonics, the maximum heat flux at the CMB would be of the same order as in the case of a stagnant lid regime $\left(\sim 100 \mathrm{~mW} \mathrm{~m}^{-2}\right.$, Nimmo and Stevenson (2000)). It is important to underline that $R a_{Q}$ has to be estimated using the superadiabatic heat flux (the total heat flux minus the adiabatic heat flux). The adiabatic heat flux for Mars' core is estimated to be in the range $5-19 \mathrm{~mW} \mathrm{~m}^{-2}$ (Nimmo and Stevenson, 2000). Using the parameter

\begin{tabular}{|c|c|}
\hline Parameters & Plausible values for Mars \\
\hline Acceleration due to gravity at the CMB, $g_{0}\left(\mathrm{~m} \mathrm{~s}^{-2}\right)$ & $\sim 3$ \\
\hline Core radius, $r_{o}(\mathrm{~km})$ & $1300-1700$ \\
\hline Density, $\rho\left(\mathrm{kg} \mathrm{m}^{-3}\right)$ & $6600-8300$ \\
\hline Thermal expansion coefficient, $\alpha\left(\mathrm{K}^{-1}\right)$ & $\sim 10^{-5}$ \\
\hline Heat capacity, $C_{p}\left(\mathrm{~J} \mathrm{~kg} \mathrm{~kg}^{-1}\right)$ & $820-860$ \\
\hline Rotation rate $($ present $), \Omega\left(\mathrm{s}^{-1}\right)$ & $7.1 \cdot 10^{-5}$ \\
\hline Kinematic viscosity, $\nu\left(\mathrm{m}^{2} \mathrm{~s}^{-1}\right)$ & $\sim 10^{-6}$ \\
\hline
\end{tabular}

Table 5: Plausible parameter values for Mars' core, after Nimmo and Stevenson (2000) and references for the first five parameters. The last parameter value is an estimation of $\nu$ in the terrestrial core.

values given in Table 5, one can estimate a plausible range of values for the maximum modified Rayleigh number $R a_{Q m}$, in Mars' core. Considering convection underneath a single plate, $R a_{Q m}$ is within the range $2 \cdot 10^{-13}-4 \cdot 10^{-13}$ whereas with a model that supposes an overturn after magma ocean crystallization (Elkins-Tanton et al., 2005), $R a_{Q m}$ is within the range $3 \cdot 10^{-12}-4.5 \cdot 10^{-12}$. These values are larger than $R a_{Q t}$. This suggests that Mars' core could have been in the hydrodynamic asymmetric regime.

In the previous section we saw that the CMB magnetic field is hemispherical in our simulations if the equatorial symmetry breaking of the flow $K_{a} / K_{s}$ is larger than 1 . The equatorial symmetry breaking which may have been due to the EAA flow component of the asymmetric regime can be roughly estimated for the past martian dynamo. Considering fixed heat flux boundary conditions, Aubert et al. (2009) have obtained a scaling law which gives the non-dimensional mean kinetic energy $K$, as a function of the dimensionless convective power $p$. In the particular case of secular cooling $p=3 / 5 R a_{Q}$ and their scaling law becomes: $K \approx 0.56 R a_{Q}^{0.84}$. Since the EAA mode results from a thermal wind mechanism, we expect the kinetic energy density related to the zonal EAA flow to be 
proportional to $R a_{Q}$ at forcings far above $R a_{Q t}$ (Aurnou et al., 2003; Aubert, 2005). Supposing that the amplitude of the meridional circulation is, at most, of the same order of magnitude as the amplitude of the zonal circulation (as it is in the first linearly unstable axisymmetric mode analytically computed by Roberts (1965) and in our nonlinear numerical simulations) then, $K_{0 a} \propto R a_{Q}$. Considering this scaling law (roughly satisfied in our numerical simulations) and the plausible values listed above for $R a_{Q m}$, we estimate that the ratio $K_{0 a} / K$ induced by the asymmetric regime would not have been larger than 0.05 in Mars' core. This result means that the EAA mode was of much weaker amplitude than the equatorially symmetric, non-axisymmetric modes and it suggests that the equatorial symmetry breaking of the flow due to the EAA mode was not large enough to induce a hemispherical dynamo in Mars' core. However such a conclusion may be hasty. First of all, we have noticed that the spontaneous emergence of the EAA mode gives birth to equatorially antisymmetric, non-axisymmetric modes as a consequence of nonlinear interactions between the EAA mode and the symmetric columnar structures. These modes might saturate with a different scaling law from the EAA mode and become of much higher amplitude than the EAA mode at planetary parameters. In such a case, the equatorial symmetry breaking might have reached higher values in Mars' core. Second, the transition between non-hemispherical and hemispherical dynamos occurs at $K_{a} / K_{s} \approx 1$ in our simulations when $P m=5$. However, there is no reason to suppose that the transition would occur at the same $K_{a} / K_{s}$ value if $P m \neq 5$. Indeed, the simulation at $P m=1$ in Fig.16(b) is the only one located above the general trend, which suggests that $P m$ may have a considerable impact on the quantitative effects of the equatorial symmetry breaking of the flow on magnetic field. Recalling that $P m$ is expected to be of the order of $10^{-6}$ in Mars' core, the transition towards hemispherical dynamos may occur at much lower $K_{a} / K_{s}$-values in planetary cores. The results obtained in Gallet and Petrelis (2009) suggest that this last point is not completely speculative: they show that even very weak equatorial symmetry breaking of the flow may lead to hemispherical dynamos. Thus, the Pm-dependence of $f_{\text {hem }}$ could be studied in order to determine if the asymmetric regime is able to explain the asymmetry of Mars' crustal magnetic field.

A heterogeneous CMB heat flux is plausible for the past martian dynamo (Stanley et al., 2008) and would make the emergence of hemispherical dynamos easier. Indeed, a strong EAA heat flux heterogeneity would directly set the amplitude of the EAA temperature contribution to $\partial T / \partial \theta$ and thus the amplitude of the EAA mode according to equation (16) (which is probably what 
fixes the amplitude of the EAA mode in the simulations of Stanley et al. (2008)). Thus, larger $K_{a} / K_{s}$-values could have been reached in Mars' core due to heterogeneous boundary conditions.

Acknowledgements: We wish to thank D. Jault and F. Pétrélis for discussions. This work was supported by program SEDIT and PNP/SEDI-TPS of French Institut National des Sciences de l'Univers (INSU), a division of CNRS. Numerical computations were performed at S-CAPAD, IPGP, France and at HPC resources from GENCI-CINES and GENCI-IDRIS (Grants 2009-042122 and 2010-042122). This is IPGP contribution 3086.

\section{References}

Acuna, M., Connerney, J., Ness, N., Lin, R., Mitchell, D., Carlson, C., McFadden, J., Anderson, K., Reme, H., Mazelle, C., Vignes, D., Wasilewski, P., Cloutier, P., 1999. Global distribution of crustal magnetization discovered by the Mars Global Surveyor MAG/ER experiment. Science 284, 790-793.

Aubert, J., 2005. Steady zonal flows in spherical shell dynamos. J. Fluid. Mech. $542,53-67$.

Aubert, J., Aurnou, J., Wicht, J., 2008. The magnetic structure of convectiondriven numerical dynamos. Geophys. J. Int. 172, 945-956.

Aubert, J., Labrosse, S., Poitou, C., 2009. Modelling the palaeo-evolution of the geodynamo. Geophys. J. Int. 179, 1414-1428.

Aurnou, J., Andreadis, S., Zhu, L., Olson, P., 2003. Experiments on convection in Earth's core tangent cylinder. Earth Plan. Sci. Lett. 212, 119-134.

Bisshopp, F., Niiler, P., 1965. Onset of convection in a rapidly rotating fluid sphere. J. Fluid. Mech. 23, 459-469.

Breuer, D., Spohn, T., 2003. Early plate tectonics versus single-plate tectonics on Mars: Evidence from magnetic field history and crust evolution. J. Geophys. Res.-Planets 108, 13.

Busse, F.H., 1970. Thermal instabilities in rapidly rotating systems. J. Fluid. Mech. 44, 441-460.

Chandrasekhar, S., 1961. Hydrodynamic and Hydromagnetic Stability. Clarendon Press, Oxford. 
Christensen, U., Aubert, J., 2006. Scaling properties of convection-driven dynamos in rotating spherical shells and application to planetary magnetic fields. Geophys. J. Int. 117, 97-114.

Christensen, U.R., Aubert, J., Busse, F.H., Cardin, P., Dormy, E., Gibbons, S., Glatzmaier, G.A., Honkura, Y., Jones, C.A., Kono, M., Matsushima, M., Sakuraba, A., Takahashi, F., Tilgner, A., Wicht, J., Zhang, K., 2001. A numerical dynamo benchmark. Phys. Earth Planet. Int. 128, 25-34.

Dormy, E., Soward, A., Jones, C., Jault, D., Cardin, P., 2004. The onset of thermal convection in rotating spherical shells. J. Fluid. Mech. 501, 43-70.

Elkins-Tanton, L., Zaranek, S., Parmentier, E., Hess, P., 2005. Early magnetic field and magmatic activity on Mars from magma ocean cumulate overturn. Earth Planet. Sci. Lett. 236, 1-12.

Ferraro, V.C.A., 1937. The non-uniform rotation of the sun and its magnetic field. Month. Not. Roy. Astr. Soc. 97, 458.

Gallet, B., Petrelis, F., 2009. From reversing to hemispherical dynamos. Phys. Rev. E 80.

Geiger, G., Busse, F., 1981. On the onset of thermal convection in slowly rotating fluid shells. Geophys. Astrophys. Fluid Dyn. 18, 147-156.

Grote, E., Busse, F., 2000. Hemispherical dynamos generated by convection in rotating spherical shells. Phys. Rev. E 62, 4457-4460.

Gubbins, D., Alfe, D., Masters, G., Price, G., Gillan, M., 2003. Can the Earth's dynamo run on heat alone? Geophys. J. Int. 155, 609-622.

Jones, C., Soward, A., Mussa, A., 2000. The onset of thermal convection in a rapidly rotating sphere. J. Fluid. Mech. 405, 157-179.

Lodders, K., Fegley, B., 1997. An oxygen isotope model for the composition of Mars. Icarus 126, 373-394.

Nimmo, F., Stevenson, D., 2000. Influence of early plate tectonics on the thermal evolution and magnetic field of Mars. J. Geophys. Res.-Planets 105, 1196911979 .

Olson, P., Christensen, U., Glatzmaier, G.A., 1999. Numerical modelling of the geodynamo: mechanisms of field generation and equilibration. J. Geophys. Res. $104,10383-10404$ 
Roberts, P.H., 1965. On the thermal instability of a highly rotating fluid sphere. Astrophys. J. 141, 240-250.

Roberts, P.H., 1968. On the thermal instability of a self gravitating fluid sphere containing heat sources. Philos. Trans. R. Soc. London, Ser. A 263, 93-117.

Sakuraba, A., Roberts, P.H., 2009. Generation of a strong magnetic field using uniform heat flux at the surface of the core. Nature Geosci. 2, 802-805.

Sanloup, C., Jambon, A., Gillet, P., 1999. A simple chondritic model of Mars. Phys. Earth Planet. Int. 112, 43-54.

Simitev, R., Busse, F., 2005. Prandtl-number dependence of convection-driven dynamos in rotating spherical fluid shells. J. Fluid. Mech. 532, 365-388.

Stanley, S., Elkins-Tanton, L., Zuber, M.T., Parmentier, E.M., 2008. Mars' paleomagnetic field as the result of a single-hemisphere dynamo. Science 321, $1822-1825$.

Stevenson, D.J., Spohn, T., Schubert, G., 1983. Magnetism and thermal evolution of the terrestrial planets. Icarus 54, 466-489.

Stewart, A.J., Schmidt, M.W., van Westrenen, W., Liebske, C., 2007. Mars: A new core-crystallization regime. Science 316, 1323-1325. 